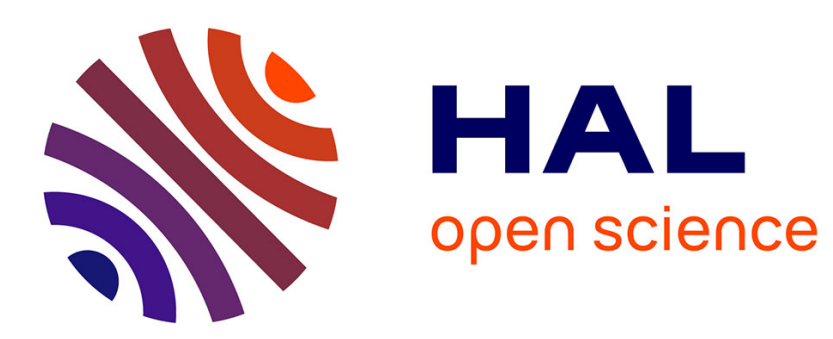

\title{
Optimized gauging for tire-rim loading identification
} K Cosseron, D Mellé, J.-F Diebold, François Hild, Stéphane Roux

\section{To cite this version:}

K Cosseron, D Mellé, J.-F Diebold, François Hild, Stéphane Roux. Optimized gauging for tirerim loading identification. European Journal of Mechanics - A/Solids, 2021, 87, pp.104192. 10.1016/j.euromechsol.2020.104192 . hal-03066228

\section{HAL Id: hal-03066228 \\ https: / hal.science/hal-03066228}

Submitted on 15 Dec 2020

HAL is a multi-disciplinary open access archive for the deposit and dissemination of scientific research documents, whether they are published or not. The documents may come from teaching and research institutions in France or abroad, or from public or private research centers.
L'archive ouverte pluridisciplinaire HAL, est destinée au dépôt et à la diffusion de documents scientifiques de niveau recherche, publiés ou non, émanant des établissements d'enseignement et de recherche français ou étrangers, des laboratoires publics ou privés. 


\title{
Optimized gauging for tire-rim loading identification
}

\author{
K. Cosseron ${ }^{a, b}$, D. Melle $e^{b, c}$, J.-F. Diebold ${ }^{b}$, F. Hild ${ }^{a}$ and S. Roux ${ }^{a}$ \\ ${ }^{a}$ Université Paris-Saclay, ENS Paris-Saclay, CNRS, LMT-Laboratoire de Mécanique et Technologie, 94235 Cachan, France \\ ${ }^{b}$ Safran Group, Safran Landing Systems, 78140 Vélizy-Villacoublay, France \\ ${ }^{c}$ now at Safran Group, Safran Tech, 78772 Magny-les-Hameaux, France
}

\section{ARTICLE INFO}

\section{Keywords:}

Optimal placement

Identification

Inverse problem

Model order reduction

Wheel

Tire

\begin{abstract}
A B S T R ACT
The determination of the tire-rim interface loadings is a difficult but key task for the aircraft wheel designer to predict the wheel service life. In conjunction with an optimal parameterization of these loadings previously defined by the authors, the optimal sensor placement problem is considered to identify the loading parameters at best. An optimization procedure of the wheel instrumentation, which consists of several strain gauges, is thus proposed to minimize the uncertainties of the sought parameters during the identification process. Two criteria are reviewed, namely, the determinant and the lowest eigenvalue of the Fisher information matrix, and different optimization procedures are assessed. The effectiveness of the method is proven considering the identification of an inflation case. The optimized instrumentations lead to drastically reduced uncertainties of the loading parameters and thus ensure reliable inverse identifications.
\end{abstract}

\section{Nomenclature}

$[A]$

$[\boldsymbol{C}]$

$[\boldsymbol{D}]$

$e_{i}\left(\left\{x_{j}\right\}\right)$

matrix describing the relationship between loading parameters and measurands covariance matrix of the uncertainties affecting the loading parameter estimates diagonal matrix whose elements $d_{i}$ are the information matrix eigenvalues

direction for a unit application of the $i$-th loading degree of freedom

$f \quad$ figure of merit

$[\boldsymbol{I}] \quad$ Fisher information matrix
$\mathcal{I}_{\varphi}$

$\mathcal{J}_{\epsilon}$

M

$N$

$\{\boldsymbol{n}\}$

$q_{i}$

$[R]$

$S$

$[\boldsymbol{V}]$

\section{$W$}

$v\left(\left\{\boldsymbol{x}_{i}\right\}\right)$

$\left\{x_{j}\right\}$

$z_{j}$

$\alpha$

lowest eigenvalue of the information matrix

cost function measuring the gap

between measured and computed strains

number of strain gauges

number of degrees of freedom

describing tire-rim interface loading

strain gauge measurement direction

amplitude of the $i$-th tire-rim interface static

eigenmode

correlation matrix of the loading parameters

surface over which potential strain gauges

may be positioned

matrix whose columns are the eigen vactors of

the information matrix

elastic strain energy

variance of the predicted quantity at point $\left\{\boldsymbol{x}_{i}\right\}$ generalized 3D coordinates of the $j$-th strain gauge location

$j$-th strain gauge measurement

orientation angle of a strain gauge

\footnotetext{
${ }^{*}$ Corresponding author

kevin.cosseron@ens-paris-saclay.fr (K. Cosseron)

ORCID(s): $0000-0001-5553-0066$ (F. Hild); 0000-0003-4885-6732 (S.
} Roux)

$\begin{array}{ll}\gamma_{\epsilon}^{2} & \text { variance of the strain measurement noise } \\ \epsilon & \text { strain tensor } \\ \omega_{j} & \text { weight associated with the } j \text {-th strain gauge } \\ \top & \text { matrix transpose } \\ \dagger & \text { Moore-Penrose pseudo-inverse }\end{array}$

\section{Introduction}

One of the key challenges to obtain predictive numerical simulations for aircraft wheel design is the knowledge of the boundary conditions prescribed by the tire during ground maneuvering. Tire-ground loads are transmitted to the wheel through the tire at the tire-rim interface. However, aircraft wheel manufacturers do not control the tire, which is a complex intermediate component. Hence, assumptions on its deformation are to be made to determine the actual loading applied to the wheel and many experiments are often required to evaluate the structural response of the wheel (Federal Aviation Administration, 2008; European Aviation Safety Agency, 2010). The knowledge of tire-rim interface loadings would thus provide an invaluable help to optimize design processes of aircraft wheels.

In recent decades, three major research topics have been proposed to tackle this challenge. First, various experimental attempts have been performed to directly measure contact pressure distributions at tire-rim interfaces using strain gauges, magnetostrictive transducers or flexible pressure sensitive films (Zaharov, 1957; Balabin and Zubarev, 1964; Walter and Kiminecz, 1975; Sherwood et al., 1995b; Wan et al., 2017). However, the intrusive nature of these measurement techniques, the fragility of the sensors and their delicate calibration raise questions about the reliability of such approaches. Second, other authors dealt with analytical (Biderman et al., 1969; Curtiss, 1973; Stearns et al., 2006; Nguyen et al., 2012; Ballo et al., 2017) or numerical (Chen, 1982; Tseng et al., 1989; Jeusette and Theves, 1992; Pelle, 1994; Chiang et al., 2000; Meng et al., 2012; 
Wan et al., 2016; Ballo et al., 2015, 2018) models of the tire. These studies are highly dependent on the material properties used for the tire model, but often consider a totally rigid wheel. For aircraft wheel manufacturers, the problem is opposite. The material properties and geometry of the wheel have to be designed, optimized and certified to sustain loads that are applied by the tire, irrespective of their specific material formulation, since different brands can be mounted on the wheel. Last, some investigations moved toward non-intrusive measurements outside the tirerim contact area (e.g., strains at gauge locations, displacements obtained via holographic interferometry) to identify loadings using inverse methods and showed promising results (Spencer et al., 1993; Kirkner et al., 1993; Schudt et al., 1994; Kandarpa et al., 1994; Spencer et al., 1995; Sherwood et al., 1995b,a; Rupp et al., 2002; Heinrietz et al., 2003).

In line with the latter research topic, the authors have proposed an inverse identification procedure of tire-rim loadings (Gras et al., 2015b; Cosseron et al., 2018) for several cases (e.g., inflation, vertical loading and cornering). The aim is to determine the loadings applied by a tire on an aircraft wheel via the measurement of the wheel deformation (e.g., displacement fields obtained by Multiview Correlation, strains at gauge locations). The underlying tasks are to define an objective parameterization of tire-rim loadings, which is as robust and compact as possible, and to choose appropriate measurements to be able to calibrate the loading parameters. A Finite Element Model Updating (FEMU) algorithm will then be used to solve this inverse problem (Kavanagh and Clough, 1971; Roux and Hild, 2020). An optimal parameterization of tire-rim interaction for aircraft wheels has been previously proposed using model reduction techniques (Cosseron et al., 2019). The static eigenmodes of the wheel stiffness matrix that is condensed at the tirerim interface are used to approximate any loading applied to the rim and cyclic symmetry properties proved to be useful to compute them. Moreover, the selection of the dominant tire-rim interface static eigenmodes, based on the elastic strain energy conservation, allowed those with the strongest impact on the structural response of the wheel to be selected. However, the identification success relies also on the measurand sensitivity to the loading parameters. A variation of the sought parameters should lead to a distinguishable variation of the measurands (i.e., greater than measurement uncertainties) in order to calibrate them accurately. Starting with a well-defined parameterization, the challenge is thus to design the experiment in such a way that the highest sensitivity to the chosen parameters is achieved.

Theoretical and practical aspects governing the general design of an experiment have been originally summarized by Fisher (1935). The author's work has been subsequently developed in the field of statistics and the concept of "optimal design of experiments" has gradually appeared in the scientific literature (Kiefer, 1959) until the reference book of Fedorov (1972). In the context of mechanical parameter identification, the optimization of an experiment consists in defining the experimental conditions to get the most accurate estimates of the unknown parameters. The optimization may deal with the loading applied during the experiment (Mehra, 1974; Morelli and Klein, 1990) as well as the placement of different types of sensors (Kammer, 1991; Wickham et al., 1995) or the geometry of the tested specimen (Schmaltz and Willner, 2014; Bertin et al., 2016). In all cases, the aim is to find the test configuration that minimizes the uncertainties of the sought parameters (Kirkegaard, 1991). Within the context of the present study, the test instrumentation is of particular interest and the aim is thus to determine the type, position and number of sensors required to get the best estimates of the sought parameters (Masroor and Zachary, 1991). However, the number of sensors is usually restricted (e.g., cost constraints, acquisition system limitations) and some sensitive areas of the structure may be inaccessible. Moreover, the addition of sensors may potentially modify the structure mechanical behavior. Taking these constraints into account, the optimization of the instrumentation is necessary to ensure $a$ priori that the test will allow the sought parameters to be calibrated at best.

Although this kind of optimization problem has been largely addressed in the literature (e.g., (Kammer, 1991; Kammer and Tinker, 2004; Meo and Zumpano, 2005; Gupta and Dhingra, 2013; Tamarozzi et al., 2016)), tire-rim interface loading identifications are usually performed with sensors located at critical stress locations on the wheel and the loading parameter uncertainties are rarely mentioned (Kandarpa et al., 1994; Heinrietz et al., 2003). Some authors have tried different configurations of strain gauges located in the immediate vicinity of the bead seat area to determine tirerim loadings (described using Fourier series), but no clear optimization procedure has been defined yet (Spencer et al., 1993; Kirkner et al., 1993; Schudt et al., 1994; Kandarpa et al., 1994; Spencer et al., 1995). This paper thus focuses on optimal sensor placement to extract maximum information about the loading parameters (i.e., tire-rim interface static eigenmode amplitudes) from experimental data and an optimization procedure is proposed to find the instrumentation that yields their most accurate estimates. The choice has been made to only deal with strain gauge measurements but the proposed framework can be easily extended to other types of sensor (e.g., camera positions for Digital Image Correlation (Vitse et al., 2020)). In conjunction with the optimal parameterization of tire-rim loadings previously defined (Cosseron et al., 2019), this approach will provide a complete answer to the problem of tire-rim loading identification.

The paper is organized as follows. Section 2 is devoted to the problem setting. The inverse problem to solve to determine tire-rim interface loadings from experimental strain measurements is presented and the challenge concerning the sensor placement is introduced. In Section 3, optimization criteria and procedures are proposed to determine an optimal instrumentation. The identification of an inflation case is then studied in Section 4 as a proof of concept. Considering a two-dimensional (2D) axisymmetric Finite Element Model (FEM) of the wheel, virtual tests are performed with 
two different tires and identification results obtained with a standard instrumentation are presented as a starting point. Finally, the instrumentation is optimized to minimize the loading parameter uncertainties.

\section{Problem setting}

A linear FEM of an aircraft wheel is considered. The loading applied by a tire on the wheel is described by $N$ degrees of freedom corresponding to the amplitudes of the dominant static eigenmodes of the wheel at the tire-rim interface (Cosseron et al., 2019). It is proposed to calibrate these loading parameters using only a few experimental strains measured by a set of gauges wisely positioned on the wheel. For each loading parameter, a unit amplitude gives rise to a strain field that is known (say from prior numerical simulation) over a surface $S$ on which potential gauges may be positioned. It is considered that an elementary gauge, centered at a point $\boldsymbol{x}$, measures the strain along one specific direction $\boldsymbol{n}$ (characterized on the free surface by an orientation angle $\alpha)$

$$
e=\boldsymbol{n} \cdot \boldsymbol{\epsilon} \cdot \boldsymbol{n}
$$

where $\boldsymbol{\epsilon}$ is the 2D strain tensor at point $\boldsymbol{x}$. The strain can thus be seen as a scalar function $e$ of the three-dimensional (3D) coordinates $(\boldsymbol{x}, \alpha)$. For the sake of simplicity, these 3D generalized coordinates will be denoted by a vector $\{\boldsymbol{x}\}$ in the following. Therefore, it is assumed that for each degree of freedom $i=1, \ldots, N$, the scalar strain field $\boldsymbol{e}_{i}(\{\boldsymbol{x}\})$ is known over the surface $S \oplus[0, \pi]$. Similarly, considering a unit amplitude of other potential loadings applied to the wheel (e.g., tie bolt tightening, axle nut tightening or nitrogen pressure), elementary strain fields associated with these loadings are available. According to the principle of superposition for a linear FEM, the strains induced on the wheel by a particular loading can thus be approximated using a linear combination of these elementary strain fields.

In order to measure the amplitudes of the $N$ degrees of freedom describing the loading applied by the tire at the tirerim interface, $M$ gauges are positioned over the surface $S$ at generalized positions $\left\{\boldsymbol{x}_{j}\right\}$ with $j=1, \ldots, M$. Each gauge is affected by a uniform uncorrelated Gaussian noise (of variance $\gamma_{\epsilon}^{2}$ ), so that the identification of the static eigenmode amplitudes gathered in the vector $\{\boldsymbol{q}\}=\left\{q_{1} \ldots q_{N}\right\}^{\top}$ are obtained from the minimization of the cost function $\mathcal{J}_{\epsilon}$ defined by

$$
\mathcal{J}_{\epsilon}=\frac{1}{\gamma_{\epsilon}^{2} M} \times \sum_{j=1}^{M}\left(z_{j}-\sum_{i=1}^{N} q_{i} e_{i}\left(\left\{\boldsymbol{x}_{j}\right\}\right)\right)^{2}
$$

and hence a set of $N$ equations is obtained by setting the gradient to zero $(\forall k \in[1, N])$

$$
\sum_{j=1}^{M} \sum_{i=1}^{N} q_{i} e_{i}\left(\left\{x_{j}\right\}\right) e_{k}\left(\left\{x_{j}\right\}\right)=\sum_{j=1}^{M} z_{j} e_{k}\left(\left\{x_{j}\right\}\right)
$$

where $z_{j}$ is the $j$-th gauge measurement from which the known contributions of tightenings and nitrogen pressure have been subtracted. Introducing the matrix $[\boldsymbol{A}]$ such that $A_{j i}=e_{i}\left(\left\{\boldsymbol{x}_{j}\right\}\right)$, Equation (3) is inverted

$$
\{\boldsymbol{q}\}=[\boldsymbol{A}]^{\dagger}\{\boldsymbol{z}\}
$$

with

$$
[\boldsymbol{A}]^{\dagger}=\left([\boldsymbol{A}]^{\top}[\boldsymbol{A}]\right)^{-1}[\boldsymbol{A}]^{\top}
$$

the Moore-Penrose pseudo-inverse matrix.

The covariance matrix of the uncertainties affecting the estimates $C_{i j}=\left\langle\delta q_{i} \delta q_{j}\right\rangle$ as induced by white Gaussian noise on the gauge measurement is thus independent of the values of the identified parameters in the linear case, and reads

$$
[\boldsymbol{C}]=\gamma_{\epsilon}^{2}[\boldsymbol{A}]^{\dagger}\left([\boldsymbol{A}]^{\dagger}\right)^{\top}=\gamma_{\epsilon}^{2}\left([\boldsymbol{A}]^{\top}[\boldsymbol{A}]\right)^{-1}
$$

Solving the inverse problem previously described allows the amplitudes of the static eigenmodes of the tire-rim interface to be determined from experimental strain measurements and their associated uncertainties to be estimated with the covariance matrix $[\boldsymbol{C}]$. It is worth noting that the proposed framework can easily be extended to other types of sensor and uncertainty of the measurements. In addition, one may ask the following questions. For a given number of gauges where (including their orientation) should one put them to get the best estimates of the sought parameters $\{\boldsymbol{q}\}$ (i.e., with the lowest uncertainties)? What is the optimal number of sensors allowing enough information about these parameters to be obtained?

\section{Optimal gauge placement}

The optimal sensor placement problem is based on the minimization of the unknown parameter uncertainties (Kirkegaard, 1991). Considering only one parameter, the problem consists in determining the sensor positions that minimize its variance. However, solving an inverse problem generally involves several parameters. Therefore, the mathematical expectation of the parameter estimates is a vector and the (co)variances are characterized by a matrix. The covariance matrix of estimated parameters is particularly relevant in that context because it deals with all facets of the inverse problem (i.e., the geometry of the studied structure, the chosen parameterization and identification methods, the boundary conditions, the measurement uncertainties). It provides an estimate of the quality of the identified parameters through their uncertainties and gives a handle on how to optimize the test (Schmaltz and Willner, 2014; Bertin et al., 2016). The minimization of the parameter uncertainties thus consists in "minimizing" the related covariance matrix. However, because the latter involves multiple dimensions, optimization criteria should be defined to recast the multi-dimensional criterion into a scalar expression that condenses the information contained in the covariance matrix (Atkinson, 1982). In this section, some optimization criteria and procedures are proposed. 


\subsection{Uncertainty minimization criteria}

The uncertainties of the identified parameters are characterized by the covariance matrix defined by Equation (6), and are independent of the parameter levels in linear cases. For a given variance $\gamma_{\epsilon}^{2}$ on the measurements, their minimization consists in modifying the matrix $[\boldsymbol{A}]$, which describes the relationship between the sought parameters and the measurands, to minimize a criterion based on the inverse of the Hessian of the least squares fit $\left([\boldsymbol{A}]^{\top}[\boldsymbol{A}]\right)^{-1}$. The work can also be equivalently carried out considering the inverse of the covariance matrix for convenience (i.e., the Fisher information matrix or Hessian). The aim is thus to maximize the information contained in the matrix $[\mathcal{I}]$, which itself will simply be

$$
[\boldsymbol{I}] \equiv[\boldsymbol{A}]^{\top}[\boldsymbol{A}]
$$

or

$$
\mathcal{I}_{i j}=\sum_{k=1}^{M} e_{i}\left(\left\{\boldsymbol{x}_{k}\right\}\right) e_{j}\left(\left\{\boldsymbol{x}_{k}\right\}\right)
$$

As a reminder, an easy way to check the relevance of a given sensor is to consider its sensitivity to the sought parameters. One may give a weight $\omega_{i}$ to each gauge $i$ (similar to the inverse uncertainty) and write

$$
\mathcal{I}_{i j}=\sum_{k=1}^{M} \omega_{k} e_{i}\left(\left\{\boldsymbol{x}_{k}\right\}\right) e_{j}\left(\left\{\boldsymbol{x}_{k}\right\}\right)
$$

The sensitivity of the $i$-th gauge is thus probed as

$$
\left[\boldsymbol{s}_{i}\right]=\frac{\partial[\mathcal{I}]}{\partial \omega_{i}}
$$

with

$$
\frac{\partial \mathcal{I}_{i j}}{\partial \omega_{k}}=e_{i}\left(\left\{\boldsymbol{x}_{k}\right\}\right) e_{j}\left(\left\{\boldsymbol{x}_{k}\right\}\right)
$$

Various criteria have been proposed to condense the information contained in the matrix $[\mathcal{I}]$ using synthetic statistics (Atkinson, 1982). Most of them are invariants of the information matrix (or its inverse) and expressed as a linear combination of its eigenvalues. For example, Mitchell (1974) used the determinant of the information matrix to minimize the volume of the uncertainties associated with the sought parameters, whereas Masroor and Zachary (1991) have chosen to study the condition number of the covariance matrix. Other authors have based their optimization on the trace of either of these matrices (Shah and Udwadia, 1977), which is equivalent to the mean variance of the identified parameters, or on the eigenvalue characterizing the direction of highest uncertainty (Bertin et al., 2016). However, the choice of the optimization criterion is difficult and varies depending on the problem being considered. In the following, two criteria are reviewed, namely, the determinant and the direction of highest uncertainty.

\subsubsection{Determinant}

One of the most used criteria is the maximization of the information matrix determinant (Mitchell, 1974). It is thus equivalent to minimizing the determinant of the covariance matrix, which represents the volume of the ellipsoid associated with parameter uncertainties. By diagonalizing the matrix $[\mathcal{I}]$ such that

$$
[\boldsymbol{I}]=[\boldsymbol{V}][\boldsymbol{D}][\boldsymbol{V}]^{\top}
$$

where $[\boldsymbol{D}]$ is a diagonal matrix gathering the eigenvalues $\left(d_{1}, \ldots, d_{N}\right)$, and $[\boldsymbol{V}]$ the matrix of the associated eigenvectors, the determinant of the information matrix reads

$$
\operatorname{det}([\boldsymbol{I}])=\operatorname{det}([\boldsymbol{D}])=\prod_{i=1}^{N} d_{i}
$$

In order to maximize it, the optimization consists in adding, removing or modifying sensor locations from an initial set. The lines $\left\{\boldsymbol{a}_{i}\right\}^{\top}$ of the matrix $[\boldsymbol{A}]$ will thus be affected. Starting with an initial instrumentation of $M$ sensors, characterized by the information matrix $\left[\boldsymbol{I}_{M}\right]$, Dykstra (1971) showed that the addition or deletion of a sensor located at the position $\left\{\boldsymbol{x}_{i}\right\}$ will modify the determinant of the new instrumentation such that

$$
\operatorname{det}\left(\left[\boldsymbol{I}_{M \pm 1}\right]\right)=\operatorname{det}\left(\left[\boldsymbol{I}_{M}\right]\right) \times\left(1 \pm v\left(\left\{\boldsymbol{x}_{i}\right\}\right)\right)
$$

where $v\left(\left\{\boldsymbol{x}_{i}\right\}\right)$ is the variance (ignoring the multiplication factor $\gamma_{\epsilon}^{2}$ ) of the predicted quantity at point $\left\{\boldsymbol{x}_{i}\right\}$ for an instrumentation composed of $M$ sensors

$$
v\left(\left\{\boldsymbol{x}_{i}\right\}\right)=\left\{\boldsymbol{a}_{i}\right\}^{\top}\left(\left[\boldsymbol{A}_{M}\right]^{\top}\left[\boldsymbol{A}_{M}\right]\right)^{-1}\left\{\boldsymbol{a}_{i}\right\}
$$

Therefore, in order to optimize the instrumentation, one will add a sensor at the location where the variance $v$ is maximum (i.e., where the lack of information is the highest) or remove the sensor where the minimum variance is reached. Kiefer and Wolfowitz (1960) have also shown the equivalence between the maximization of the information matrix determinant (D-optimality) and the minimization of the maximum variance over the predicted quantities (G-optimality). One may also perform an exchange between a sensor from the initial instrumentation and another one located at positions $\left\{\boldsymbol{x}_{i}\right\}$ and $\left\{\boldsymbol{x}_{j}\right\}$ respectively (Fedorov, 1972). In that case, the determinant for the new configuration, characterized by the information matrix $\left[\boldsymbol{I}_{M^{\prime}}\right]$, reads

$$
\operatorname{det}\left(\left[\boldsymbol{I}_{M^{\prime}}\right]\right)=\operatorname{det}\left(\left[\boldsymbol{I}_{M}\right]\right) \times\left(1+\Delta\left(\left\{\boldsymbol{x}_{i}\right\},\left\{\boldsymbol{x}_{j}\right\}\right)\right)
$$

with

$$
\begin{aligned}
\Delta\left(\left\{\boldsymbol{x}_{i}\right\},\left\{\boldsymbol{x}_{j}\right\}\right)= & v\left(\left\{\boldsymbol{x}_{j}\right\}\right)-\left[v\left(\left\{\boldsymbol{x}_{i}\right\}\right) v\left(\left\{\boldsymbol{x}_{j}\right\}\right)\right. \\
& \left.-v\left(\left\{\boldsymbol{x}_{i}\right\},\left\{\boldsymbol{x}_{j}\right\}\right)^{2}\right]-v\left(\left\{\boldsymbol{x}_{i}\right\}\right)
\end{aligned}
$$

and the optimization consists in exchanging the sensor pair associated with the maximum value of $\Delta$. Furthermore, it is worth noting that the use of the determinant as the optimization criterion requires the update of the information matrix at each modification of the instrumentation. 


\subsubsection{Direction of highest uncertainty}

Another possible criterion is to consider the eigenvalue of the covariance matrix associated with the direction of highest uncertainty (i.e., the length of the largest semi-major axis of the uncertainty ellipsoid) and requires to minimize it. It thus consists in maximizing the lowest eigenvalue of the information matrix. By denoting $\{\varphi\}$ the considered direction in the parameter space, the criterion aims at maximizing the quantity $\mathcal{I}_{\varphi}$ defined by

$$
\mathcal{I}_{\varphi}=\{\boldsymbol{\varphi}\}^{\top}[\boldsymbol{I}]\{\boldsymbol{\varphi}\}=\sum_{k} \omega_{k} f\left(\left\{\boldsymbol{x}_{k}\right\}\right)^{2}
$$

with

$$
f\left(\left\{\boldsymbol{x}_{k}\right\}\right)=\sum_{i} \varphi_{i} e_{i}\left(\left\{\boldsymbol{x}_{k}\right\}\right)
$$

for a given number of measurements $\sum_{k} \omega_{k}$ (with $\omega_{k} \leq 1$ to account for the fact that two gauges cannot overlap). In the continuum, one can progressively remove the weight $\omega$ given to sensors located in the regions where $f^{2}$ is minimum, update the information matrix and recompute the direction of highest uncertainty. For a fixed direction $\{\varphi\}$, the solution is simply to threshold the regions where $f^{2}$ is highest.

\subsection{Optimization procedures}

To determine the optimal placement of $M$ sensors over a potential surface $S$ of the studied structure, the above criteria should be maximized. One possibility is to start with a large population of $M_{\text {tot }}$ possible gauges and progressively remove the least useful until the number $M$ of desired sensors is reached. One may also consider the complementary procedure, which consists in successively adding gauges from an initial configuration. For a single direction criterion, the choice is trivial since all gauges have a well-defined figure of merit $f^{2}$. The point where this field is maximum should host the new gauge whereas the one where it is minimum should be removed. Similarly, one may decide to move gauges along $\nabla\left(f^{2}\right)$ rather than using the above quantum version with creation/annihilation operators. For the criterion based on the direction of highest uncertainty, the same situation as above is retrieved but the eigen direction $\{\boldsymbol{\varphi}\}$ has to be updated at each iteration. For the determinant criterion, the same philosophy holds considering the variance $v$ associated with each gauge.

With these two processes at hand, it is possible to alternatively add and delete one gauge to drive the population to a satisfactory distribution. The procedures based on this principle are known as exchange algorithms. The first step to use them is to choose an initial set of $M$ gauges from a prespecified list of $M_{t o t}$ possible ones. The sequential exchange algorithm (Wynn, 1970; Dykstra, 1971) then switches between the addition and deletion of a gauge at each iteration until convergence of the chosen criterion. An $(M+1)$-th gauge is first added so that the maximum increase of the chosen criterion is achieved and the one of the new configuration resulting in the minimum decrease is then removed.
The operations may equivalently be performed in the reverse order (Van Schalkwyk, 1971).

It is also possible to perform a direct exchange between a gauge pair at each iteration, in particular when the information matrix determinant is used as the optimization criterion. The Fedorov (1972) algorithm reviews all the possible exchange pairs between the $M$ sensors of the initial configuration and the $M_{r}=M_{t o t}-M$ remaining candidates and proceeds to the exchange yielding the maximum value of $\Delta$ defined by Equation (17). The information matrix is then updated and the process is repeated until convergence of the optimization criterion. However, this algorithm is relatively slow because it requires $M \times M_{r}$ comparisons at each iteration to finally perform only one exchange. Cook and Nachtsheim (1980) proposed a modified Fedorov algorithm, faster than the original one, within which each iteration is subdivided into $M$ steps. At a given iteration, the first gauge of the current configuration is selected and the exchange pairs between this specific gauge and the $M_{r}$ candidates are studied. The pair that maximizes the criterion is exchanged, the information matrix is updated and the operation is repeated for the $M-1$ remaining gauges. This algorithm thus allows $M$ exchanges to be performed at each iteration.

Another possibility to speed up the procedure is to exchange only the $K$ least useful gauges at each iteration. This scheme is known as the $K$-exchange algorithm (Galil and Kiefer, 1980; Johnson and Nachtsheim, 1983). For $K=1$ (resp. $K=M$ ), the above described sequential exchange algorithm (resp. modified Fedorov algorithm) is retrieved. However, the choice of the number $K$ is not trivial and often depends on the problem being considered (Meyer and Nachtsheim, 1995). An enhanced version of this scheme, namely the $K L$-exchange algorithm, was also proposed by Atkinson and Donev (1989). The idea is to only exchange the $K$ least useful gauges at each iteration considering only a list of $L \leq M_{r}$ candidates.

All the above mentioned algorithms correspond to nonlinear optimization techniques and there is no guarantee that the global optimum will be reached. However, the approach is somewhat unavoidable (due to the discrete nature of the problem and its lack of convexity) and they reveal very effective in practice. To ensure that the solution is in the neighborhood of the global optimum, several initializations should be tested to check their stability. Furthermore, one of the drawbacks of these approaches is the dependence to the considered model, the optimization being conducted to obtain its most accurate estimate. Therefore, a modification in the parameterization is likely to yield different optimal instrumentations. DuMouchel and Jones (1994) proposed a Bayesian modification of the previous algorithms to reduce the dependence on an assumed model. The sensor placement obtained with it allows the sought parameters to be calibrated at best but also to take into account the effect of additional and higher order parameters not included in the initial model. 


\section{Identification of an inflation case}

In this section, it is proposed to determine the optimal placement of a given number of gauges to identify at best the loading applied by a tire on an aircraft wheel during an inflation case. First, a 2D-axisymmetric FEM of the wheel is presented, as well as the loading parameterization. Then, virtual tests are performed considering two different tires. Last, the identification results obtained with standard and optimized instrumentations are presented.

\subsection{Modeling}

\subsubsection{Finite element model of the wheel}

Owing to the nature of the loading to be determined, a 2D-axisymmetric linear FEM of an aircraft wheel has been developed with the commercial software ANSYS Mechanical 16.2 (Figure 1). It only contains two wheel halves (inboard and outboard) and the assumption is made that a perfect connection exists between the two parts. The tie bolts joining the halves are thus not modeled. The material properties are those of an aluminum alloy with a linear elastic behavior. The mesh contains 2,500 quadratic and axisymmetric elements and 5,700 nodes. Boundaries are clamped on bearing support surfaces, and the loadings applied to the wheel are the nitrogen pressure and the tire-rim contact tractions.

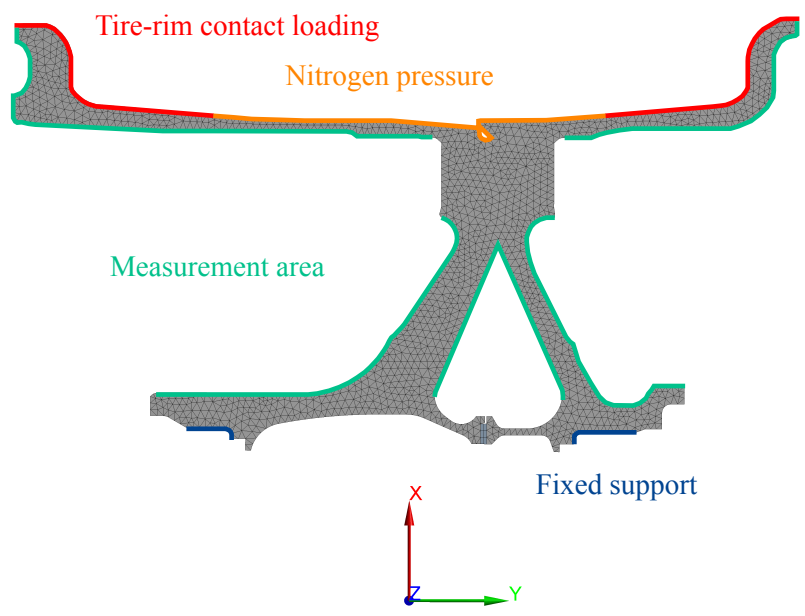

Figure 1: 2D-axisymmetric FEM of the wheel

Strain gauges are positioned over the surface $S$ of the wheel (highlighted in turquoise in Figure 1) to calibrate the loadings at the tire-rim interface. It has to be noted that the tire-rim interface itself (highlighted in red in Figure 1) has been deliberately excluded from this measurement area because of its delicate environment. The sensors may be damaged because of shear forces encountered in the contact area during tire-wheel assembly. The nitrogen pressure area is not included as well due to the specific post-processing of the measured strains required in that zone (Jansen, 1997). Last, the assembly area is not taken into account because of its simplification.

\subsubsection{Static eigenmodes of tire-rim interface}

It is chosen to identify the amplitudes of the static eigenmodes of the tire-rim interface that are used to approximate the loading applied to the wheel by the tire. These modes were obtained using the methodology described by Cosseron et al. (2019). To summarize, the stiffness matrix of the wheel is first condensed at the tire-rim interface (i.e., Guyan reduction (Guyan, 1965)), and the resulting reduced matrix is then diagonalized to obtain a complete set of generalized shape functions (i.e., modes) for describing the tire-rim interface displacement. The deformed shape of the wheel due to the unit application of the first eight static eigenmodes of the tire-rim interface, ordered by increasing eigenvalue, are illustrated in Figures 2 and 3.

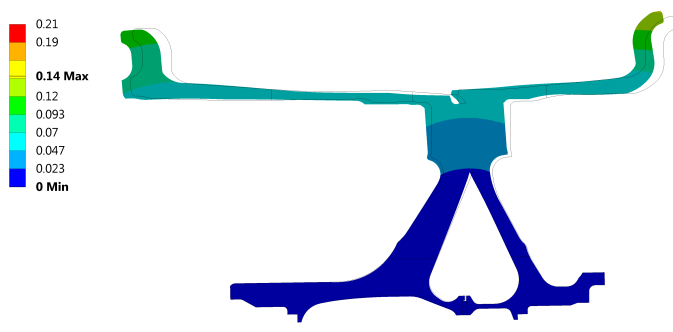

(a)

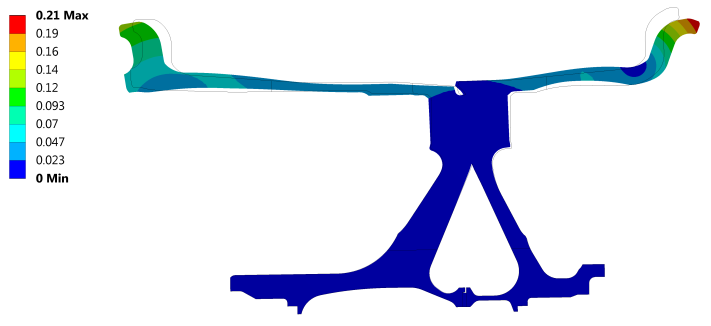

(b)

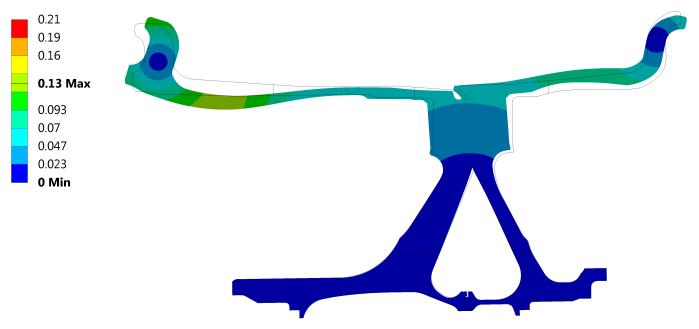

(c)

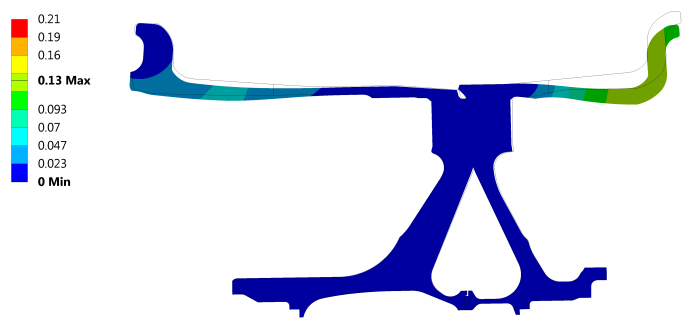

(d)

Figure 2: Deformed shape of the wheel for the first eight tirerim interface static eigenmodes (magnification factor $\times 100$ ) Modes 1 (a) to 4 (d) - Displacement norm ( $\mathrm{mm}$ ) 


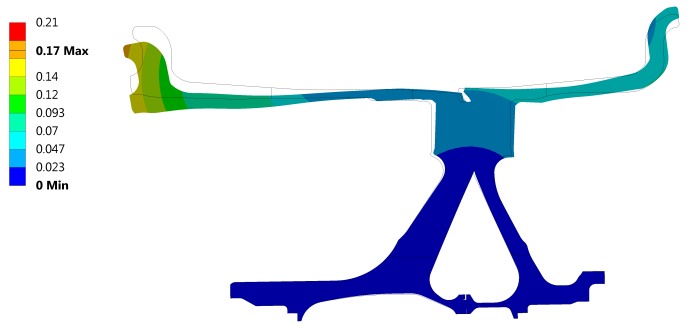

(a)

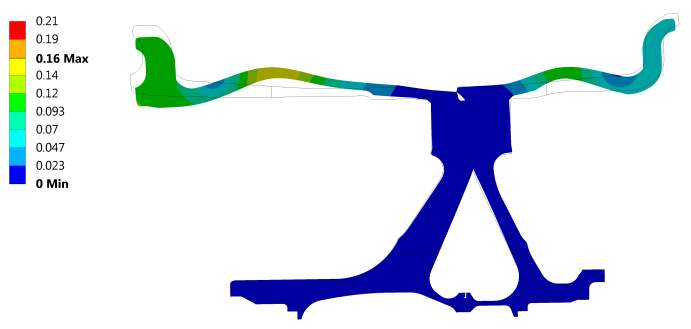

(b)

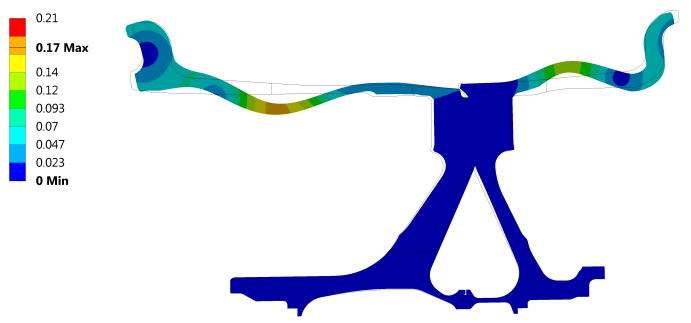

(c)

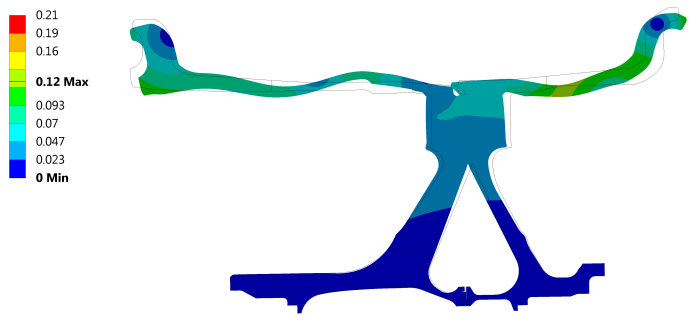

(d)

Figure 3: Deformed shape of the wheel for the first eight tirerim interface static eigenmodes (magnification factor $\times 100$ ) Modes 5 (a) to 8 (d) - Displacement norm ( $\mathrm{mm})$

The first modes display global responses. The deformed shapes induced by modes 1 and 2 are essentially axial, whereas they are mainly radial on the inboard (resp. outboard) side of the wheel for mode 3 (resp. 4). For higherorder modes, the deformation only affects a small confined region of the rim. Furthermore, a side study showed that low-order eigenmodes were not very sensitive to the mesh size and were thus deemed reliable.

The eigenmode basis retains the initial number of tirerim interface degrees of freedom and only corresponds to a different representation of the tire-rim loading (i.e., basis change) with no particular assumptions. The mathematical problem is thus strictly equivalent but easier to handle because of the diagonalization of the wheel stiffness matrix that is condensed at the tire-rim interface. To limit the number of loading parameters to be calibrated, a criterion based on the elastic strain energy conservation is used to select the dominant eigenmodes required to approximate a given static loading and will be explained in the next section.

\subsection{Virtual tests \\ 4.2.1. Available data}

Tire manufacturers usually give maps of the contact pressure distribution at the tire-rim interface for different loading cases to support aircraft wheel manufacturers in the development of their products. They are obtained using finite element analyses or experiments, yet the wheel is considered totally rigid in both cases, which is a strong assumption for the aircraft wheel manufacturer. However, these data are used to evaluate the structural response of the wheel before performing validation tests and are thus very useful for the aircraft wheel designer.

In the following, two radial tires (designated A and B) that can be mounted on the studied wheel are investigated. The tire-rim contact pressure distributions are given by tire manufacturers for an inflation pressure of 14.8 bar for tire A, and 14.7 bar for tire B. They are plotted against the curvilinear abscissa along the rim profile in Figure 4.

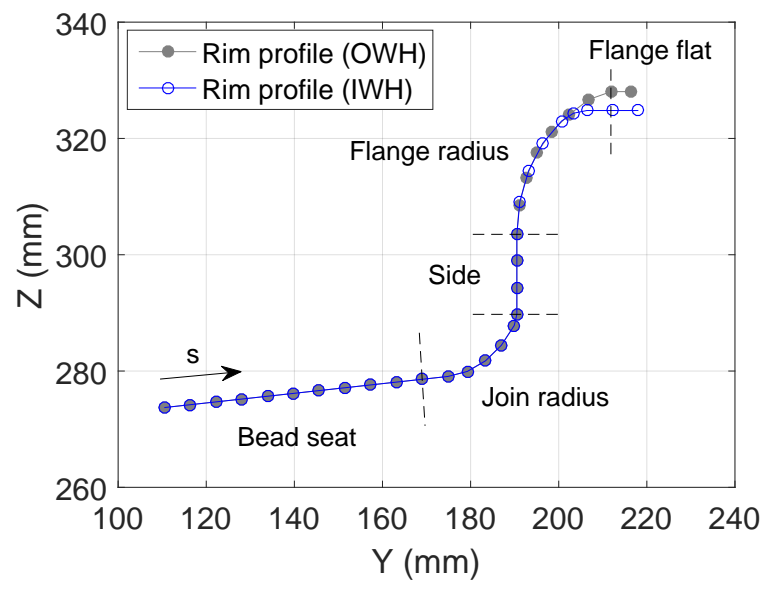

(a)

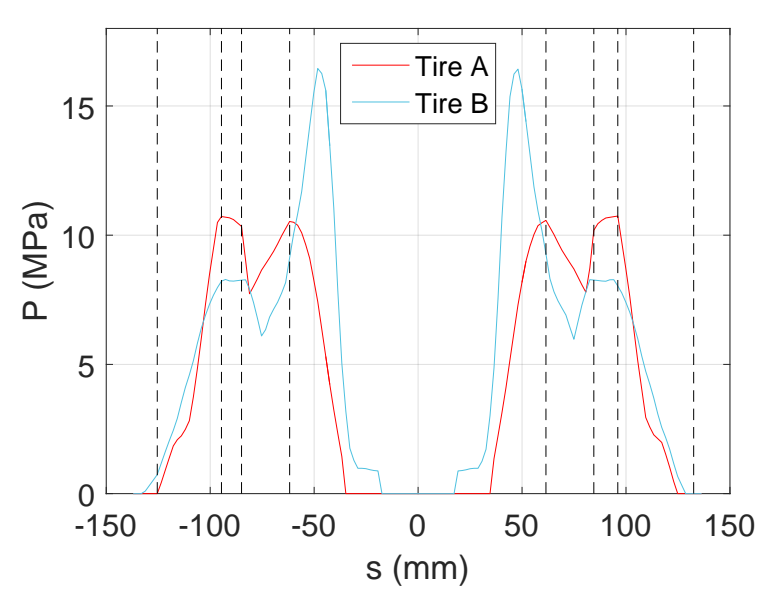

(b)

Figure 4: Available data for inflation case of tires $A$ and $B$. Rim profile (a). Tire-rim contact pressure distributions (b) 
By convention, the curvilinear abscissa is negative (resp. positive) for the inner (resp. outer) wheel half, and its origin is located, for each side, at the point of the tire-rim contact area nearest to the wheel center, as shown in Figure 4a. Dashed lines are added to depict characteristic areas of the rim profile, such as the bead seat or the flange. Despite the fact that the inflation pressures are close, the two tires exhibit very different distributions (Figure 4b). A balanced pressure distribution between the bead seat and the flange is observed for tire A, whereas the pressure is higher in the bead seat area for tire B.

It is proposed to perform virtual tests using the tire-rim interface loadings given by manufacturers for the two tires, and then carry out inverse identifications of the loading parameters based on virtual strain measurements on the wheel. Therefore, tire-rim contact pressure distributions shown in Figure $4 \mathrm{~b}$ are prescribed in the 2D-axisymmetric FEM of the wheel (Figure 1), as well as the corresponding nitrogen pressure. The deformed shapes of the wheel induced by each tire inflation are illustrated in Figure 5.
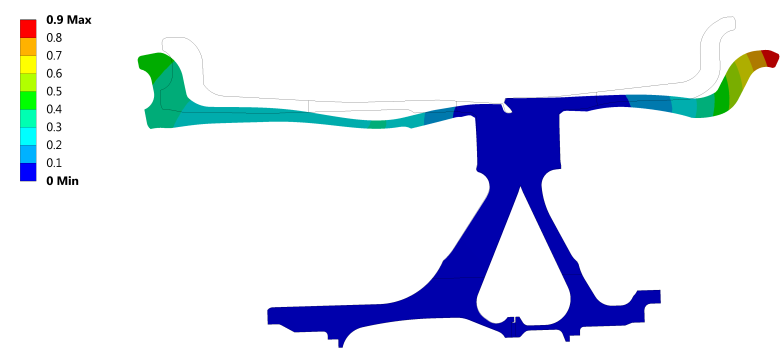

(a)
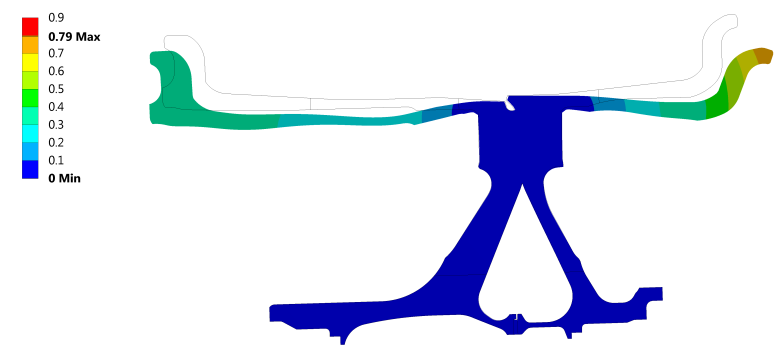

(b)

Figure 5: Deformed shape of the wheel for inflation case of tires A (a) and B (b) (magnification factor $\times 50$ ) - Displacement norm $(\mathrm{mm})$

The radial displacement in the bead seat area is higher for tire B whereas bending of the flanges is more pronounced for tire A. This observation is consistent with the differences observed in the tire-rim contact pressure distributions (Figure $4 \mathrm{~b}$ ).

The strains induced by each tire inflation were postprocessed over the measurement area highlighted in Figure 1 to serve as virtual measurements for tire-rim loading identifications. It has to be noted that strains are read at each potential gauge center and not averaged over the gauge surface. Moreover, no noise is added to these raw data.

\subsubsection{Selection of the loading parameters}

Any loading applied to the wheel by a tire is described using the static eigenmodes mentioned earlier. Therefore, their amplitudes will be sought in future inverse identifications. However, the number of parameters to be identified is crucial and should be minimized. A selection criterion based on the elastic strain energy conservation (Cosseron et al., 2019) is thus used to reduce the number of selected modes to a minimum. The tire-rim contact pressure distributions of tires $\mathrm{A}$ and $\mathrm{B}$ are first converted into nodal equivalent forces and projected onto the tire-rim interface static eigenmode basis. A specific script was developed to this end using $M A T L A B^{\circledR}$. Normal pressures are converted into cylindrical pressures by computing the rim profile angle at each node of the rim mesh and nodal equivalent forces in radial and axial directions are then obtained by multiplying the corresponding pressures with the elementary area associated with each node. The elastic strain energy distribution over the modes for each tire inflation is then computed and illustrated in Figure 6, where eigenmodes are ranked with increasing eigenvalue. Only the first 30 modes, among approximately 300 , are reported to focus on data of interest.

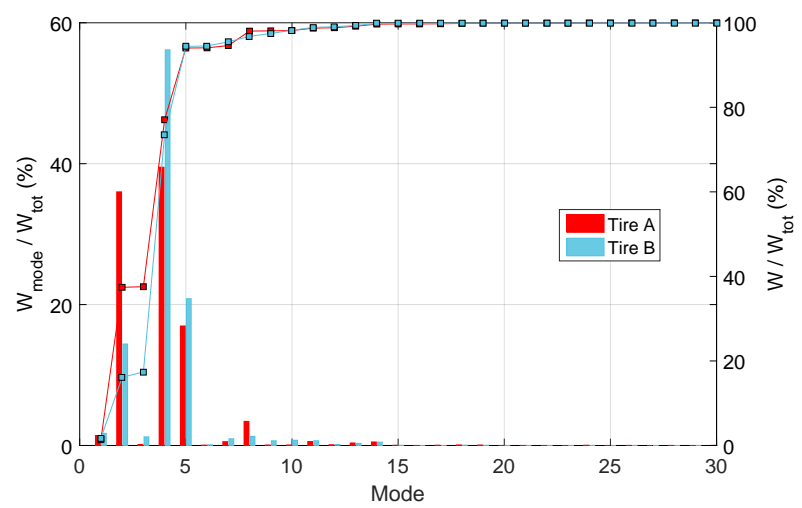

Figure 6: Elastic strain energy contribution of the tire-rim interface static eigenmodes for inflation case of tires $A$ and $B$

The excited modes are globally the same for inflation cases of the two tires and both are low-order ones. To assess how many of them are required to effectively approximate either of these loadings, they are ranked in descending order of elastic strain energy contribution for each tire. The resulting ranking is reported in Table 1. To keep 99\% of the total elastic strain energy, 13 (resp. 11) modes are required to approximate the inflation case of tire A (resp. B). The rankings are not exactly the same for the both tires but, among the 13 modes required for tire A, 12 are strictly identical to the first dominant modes required for tire $\mathrm{B}$. The inflation loadings applied to the wheel by the two tires can thus be described using a reduced common set of 12 modes (highlighted in bold in Table 1). The choice of the truncation criterion value (i.e., $99 \%$ of the total elastic strain energy) will be carefully discussed in the following. 
Table 1

Dominant eigenmodes for inflation case of tires $A$ and $B$

\begin{tabular}{cc}
\hline Tire & Dominant eigenmode number \\
\hline A & $\mathbf{4 - 2 - 5 - 8 - 1 - 1 1 - 7 - 1 4 - 1 3 - 3 - 1 2 - 1 8 - 1 0 - . . .}$ \\
B & $\mathbf{4 - 5 - 2 - 1 - 8 - 3 - 7 - 1 0 - 1 1 - 9 - 1 4 - 1 3 - 1 2 - . . .}$ \\
\hline
\end{tabular}

Therefore, it is reasonable to assume that any inflation loading applied to the rim could be described with this particular set of modes. Their amplitudes will be sought in future inverse identifications because of their potential identifiability. These modes are numbered from 1 to 12 and ordered by increasing eigenvalue in the following. The reference values of these 12 amplitudes, obtained by simple projection of tire-rim loadings on the eigenmode basis, which are to be retrieved, are depicted in Figure 7. Amplitudes of the first modes are naturally higher than those of the last ones. However, they allow the two studied tires to be distinguished.

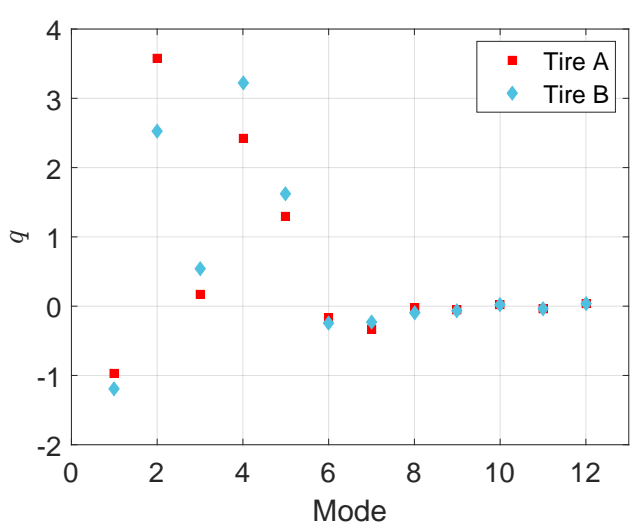

Figure 7: Reference modal amplitudes for inflation case of tires $A$ and $B$

\subsection{Results}

\subsubsection{Standard instrumentation}

A standard instrumentation, which is commonly used during the validation process of an aircraft wheel, is first considered to determine the inflation loadings of tires A and B. This instrumentation is illustrated in Figure 8.

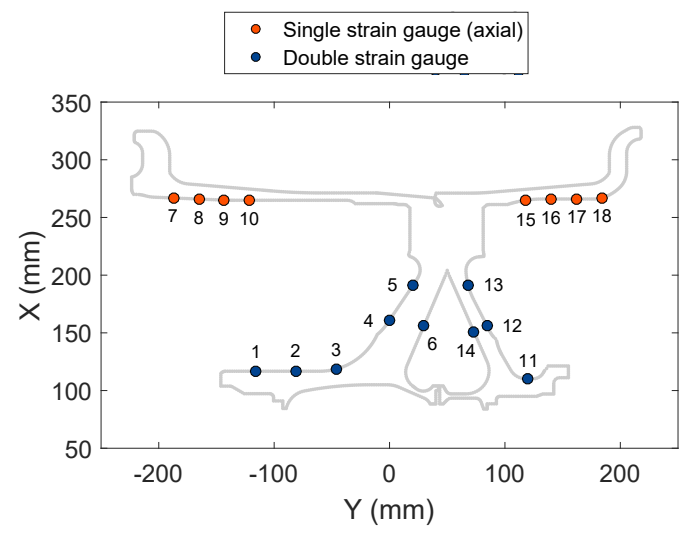

Figure 8: Standard instrumentation
It contains 18 strain gauges, including 10 double rosettes and 8 single gauges, for a total of $M=28$ strain measurements. For each gauge, the axial (resp. circumferential or hoop) measurement direction is defined by the tangent to the wheel in the $X-Y$ (resp. $X-Z$ ) plane at the considered gauge center. The virtual strain measurements at gauge locations obtained for the inflation cases of tires A and B (with no added noise) are reported in Figure 9 and confirm their differences in behavior.

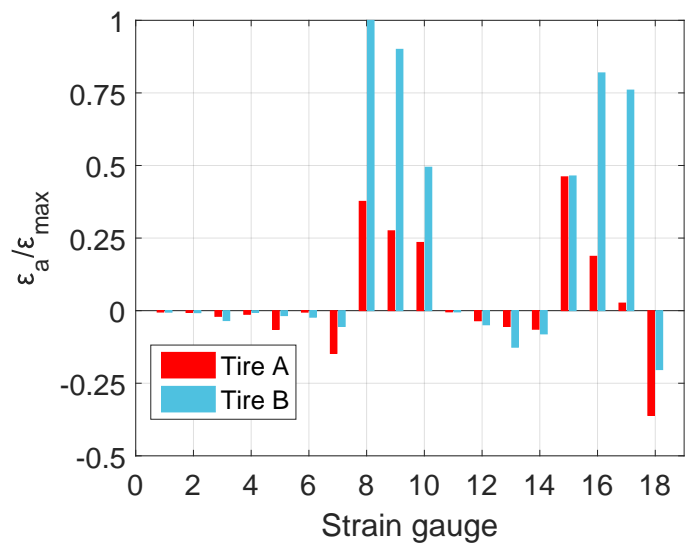

(a)

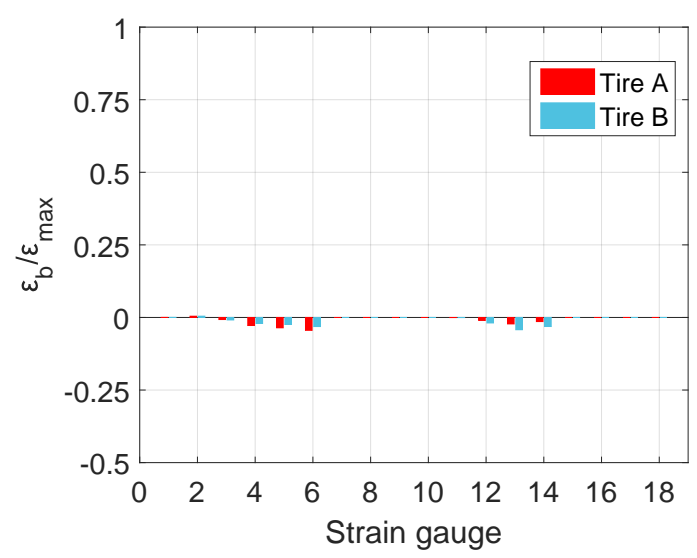

(b)

Figure 9: Virtual strain measurements at gauge locations for inflation case of tires A and B. Axial (a) and hoop (b) strains

It has to be noted that the considered instrumentation has not been designed specifically to calibrate loadings at the tire-rim interface. The placement of the different gauges was chosen after preliminary finite element analyses and subsequent location of the critical areas of the wheel, as classically carried out in mechanical design offices. However, one may wonder whether these measurements are sufficient to calibrate the 12 modal amplitudes. To this end, the matrix [A] defined in Section 2 is first computed. Each column is obtained by prescribing a unit amplitude to one of the 12 static eigenmodes in the 2D-axisymmetric FEM of the wheel and post-processing the induced strains at gauge locations for each considered direction. The matrix $[\boldsymbol{A}]$ obtained for the standard instrumentation is illustrated in Figure 10 and con- 
tains $M=28$ rows and $N=12$ columns.

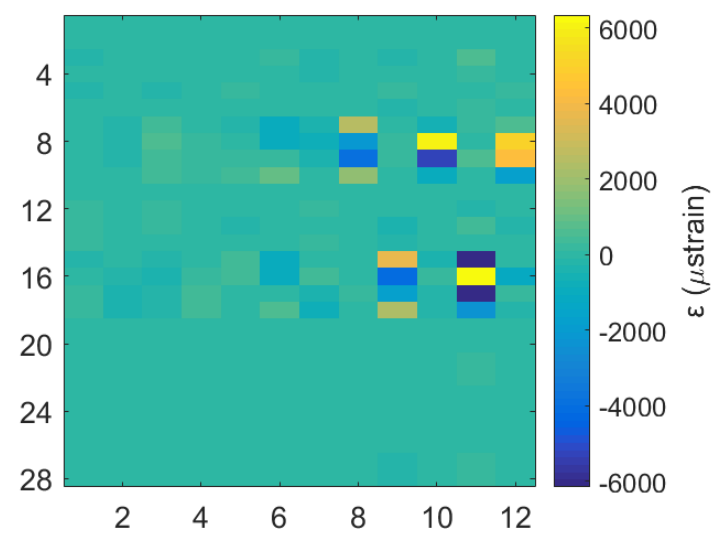

Figure 10: Matrix $[\boldsymbol{A}]$ for the standard instrumentation

The first (resp. last) rows correspond to strains measured in the axial (resp. hoop) direction of the considered gauges. The strain levels induced by the first modes are lower than those of the last ones because of the mode ranking by increasing eigenvalue. By considering that the uncertainty of the strain measurements has a standard deviation $\gamma_{\varepsilon}=10 \mu$ strain, the covariance matrix of the sought parameters is computed using Equation (6) even before running the identification procedure itself (Figure 11).

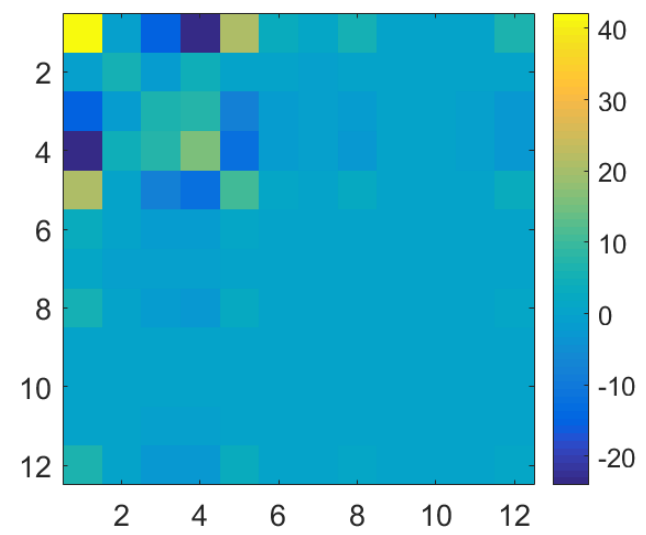

Figure 11: Covariance matrix $[\boldsymbol{C}]$ for the standard instrumentation

The diagonal terms of the covariance matrix, which correspond to the variances of the parameter estimates when they are independently considered (or $\gamma_{k}=\sqrt{C_{k k}}$ ), show that uncertainties are particularly high for the first five eigenmode amplitudes. Whatever the identification results, the calibrated parameters will thus be unreliable. To highlight this point, the inverse problem is solved using Equation (4) for the two "virtual tests" and the calibrated modal amplitudes are compared to the references in Figure 12. The error bars indicate the standard deviations $\gamma_{k}$ for each loading parameter.

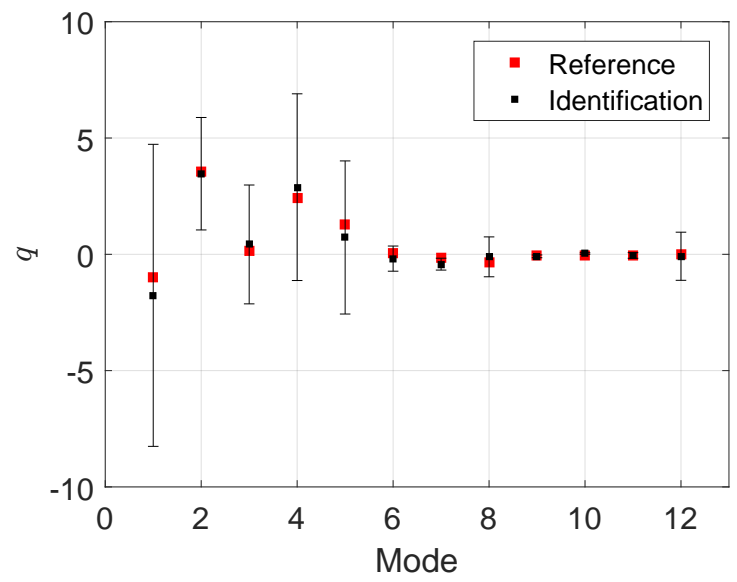

(a)

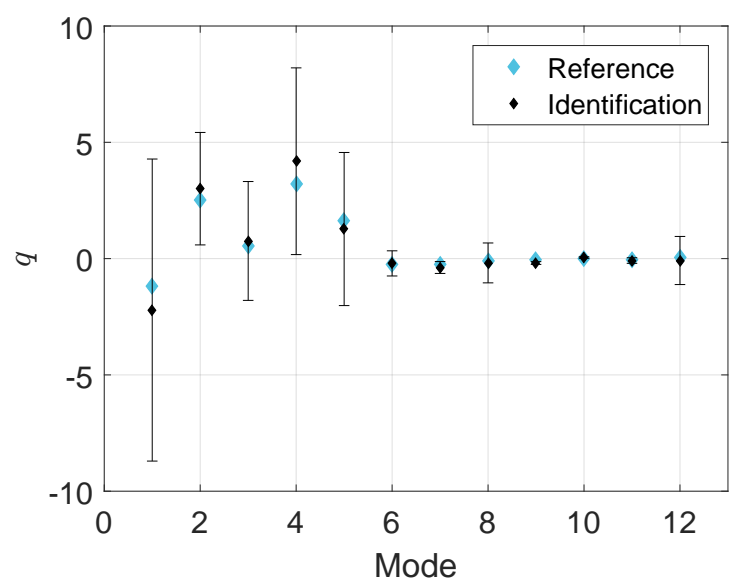

(b)

Figure 12: Comparison between the reference and calibrated modal amplitudes for the inflation case of tires $A(a)$ and $B$ (b) using a standard instrumentation

The identified modal amplitudes are very close to their reference values. One may think that the standard instrumentation is thus suitable to correctly calibrate the sought parameters. However, these results are explained by the fact that the same model is used to build the inverse problem and to generate the virtual strain measurements, the differences being strictly limited to the truncation of the eigenmode basis. This phenomenon - called the "inverse crime" in the literature (Kaipio and Somersalo, 2005) — leads to over-optimistic results compared to real test measurements. It should be recalled that no noise was added to the virtual strain measurements. Therefore, the small differences between reference and identified modal amplitudes originate solely from the truncation of the eigenmode basis to the first 12 dominant modes. This truncation gives a parameterization to approximate tire-rim interface loadings that, when fed to the inverse problem, naturally produces a discrepancy between reference and identified modal amplitudes.

However, the loading parameter uncertainties (i.e., error bars in Figure 12) show that the results are clearly not satisfactory. If the virtual strain measurements were slightly 
disturbed (e.g., adding noise of standard deviation $\gamma_{\varepsilon}=$ $10 \mu$ strain), the calibrated modal amplitudes will be very different and farther away from their nominal values because the problem is ill-conditioned. For the considered instrumentation, this observation indicates that the error due the truncation of the eigenmode basis is less harmful than the measurement noise uncertainty with the variance chosen here, even though the two are strongly correlated. Furthermore, one can notice that the covariance matrix is not diagonal due to the presence of couplings between the parameters. Another indicator to visualize them is the correlation matrix $[\boldsymbol{R}]$ defined as

$$
R_{i j}=\frac{C_{i j}}{\sqrt{C_{i i} C_{j j}}}
$$

The diagonal terms are equal to 1 because each parameter is perfeclty correlated to itself, whereas the off-diagonal terms range from -1 to 1 , and indicate the linear dependence (i.e., cross-correlation) between two parameters. The correlation matrix obtained for the standard instrumentation is shown in Figure 13.

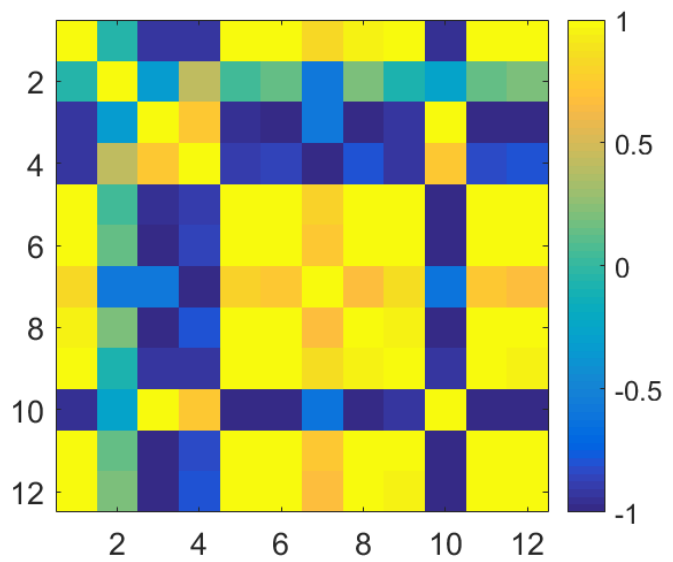

Figure 13: Correlation matrix $[\boldsymbol{R}]$ for a standard instrumentation

The loading parameters are highly correlated (or anticorrelated) and the correlations for each parameter pairs characterize the inclination of the uncertainty ellipsoid in the parameter space. Therefore, the parameters cannot be considered separately, and the standard deviations $\gamma_{k}$ mentioned earlier are no longer suitable to fully account for the uncertainties. The consideration of the principal directions of the uncertainty ellipsoid is thus necessary to take into account the fluctuations of all the parameters (Gras et al., 2015c). The diagonalization of the information matrix using Equation (12) gives the variance of the $k$-th eigenvector amplitude

$$
\tau_{k}^{2}=\frac{\gamma_{\varepsilon}^{2}}{D_{k k}}
$$

and the standard deviation $\tau_{k}$ characterize the "length" of the $k$-th semi-major axis of the uncertainty ellipsoid. To summarize, the standard instrumentation presented in Figure 8 is not appropriate for the calibration of the sought loading parameters because of the large resulting uncertainties. In the following, it is thus proposed to define an optimal instrumentation based on the optimization criteria and procedures discussed in Section 3.

\subsubsection{Optimized instrumentations}

A set of 148 double strain gauges is considered to define an optimal instrumentation and to calibrate the 12 modal amplitudes at best (Figure 14). These gauges form a total of $M_{\text {tot }}=296$ possible strain measurements and cover all the measurement area presented in Figure 1.

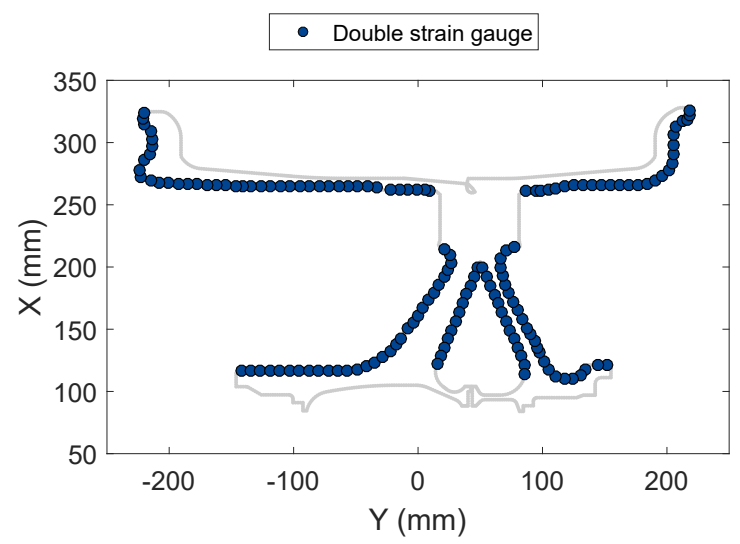

Figure 14: Location of the 148 potential gauges for the calibration of the loading eigenmodes

It is proposed to perform several optimizations of the instrumentation considering the two criteria described in Section 3, namely, the information matrix determinant and the direction of highest uncertainty. The results obtained for each criterion are presented successively and several optimization algorithms are assessed. In all cases, a minimum of $M=12$ strain measurements is kept to calibrate the 12 loading parameters.

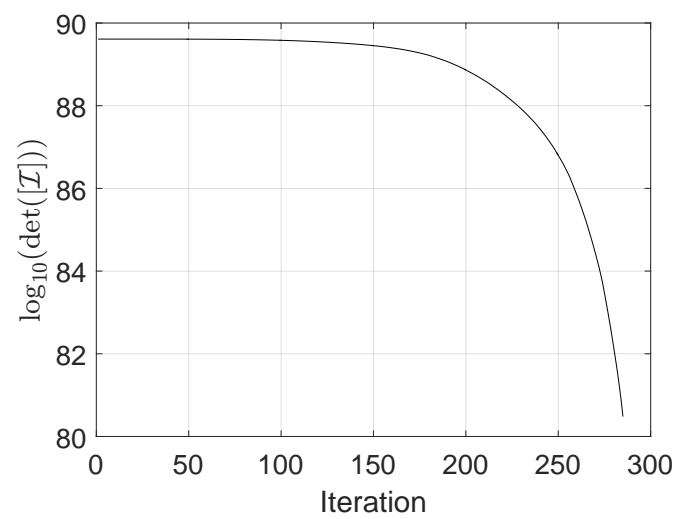

Figure 15: Determinant criterion change with the number of iterations 
The simplest algorithm to maximize the information matrix determinant, for a given number of gauges, is to start with the $M_{t o t}=296$ possible measurements (Figure 14) and progressively remove the one for which the variance $v$ defined by Equation 15 is minimum. The decrease of the determinant criterion is illustrated in Figure 15 as a function of the iteration number. The computation takes only a few seconds due to the size and linearity of the FEM.

When the algorithm is stopped to retain only $M=12$ strain measurements, the instrumentation illustrated in Figure 16 is obtained. All the gauges required for the loading recovery are located on the rim of the wheel. This result was predictable because of the sensitivity of this area to the local distribution of the tire-rim interface loading. However, one can note that the gauges are uniformly distributed over the rim. Moreover, there are only single strain gauges measuring in the axial direction, except for the one at the tip of the outer wheel half.

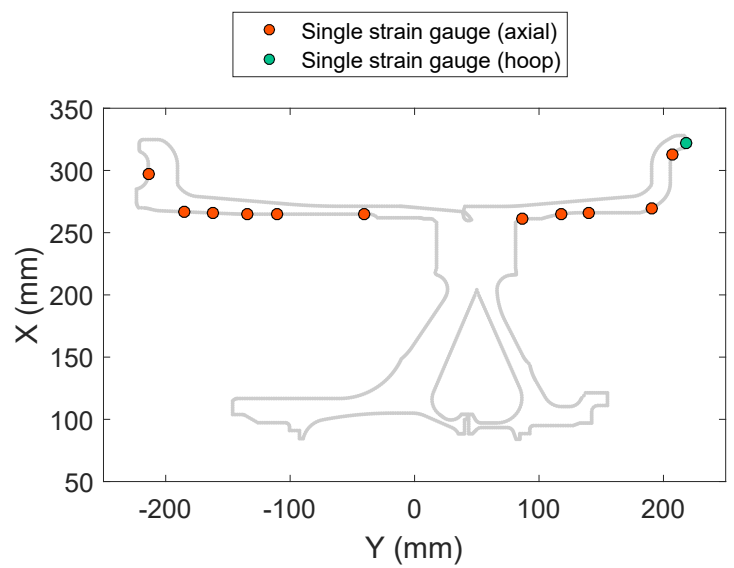

Figure 16: Locations of the 12 retained strain gauges for optimized instrumentation \#1

The covariance and correlation matrices for this new instrumentation are gathered in Figure 17. The uncertainties of the sought parameters were substantially reduced in comparison with the standard instrumentation (Figure 11). Moreover, most of the couplings between the parameters were removed. All off-diagonal terms of the correlation matrix are now less than or equal to 0.5 .

Further, the inverse problem is solved considering that strain measurements at optimized gauge locations are available for the two virtual inflation tests. The modal amplitudes are compared to their reference values in Figure 18. The differences are very small, and each modal amplitude is obtained with a high degree of accuracy. The error bars are almost invisible due to their small size. In contrast to the standard instrumentation case, the truncation error here is much larger than the random uncertainty. The quality of the gauges and their placement motivate a better representation of the tire-rim loading. After considering the effect of noise, one can thus point out the truncation order (initially chosen to keep $99 \%$ of the total elastic strain energy) such that it induces an error less than that of noise.

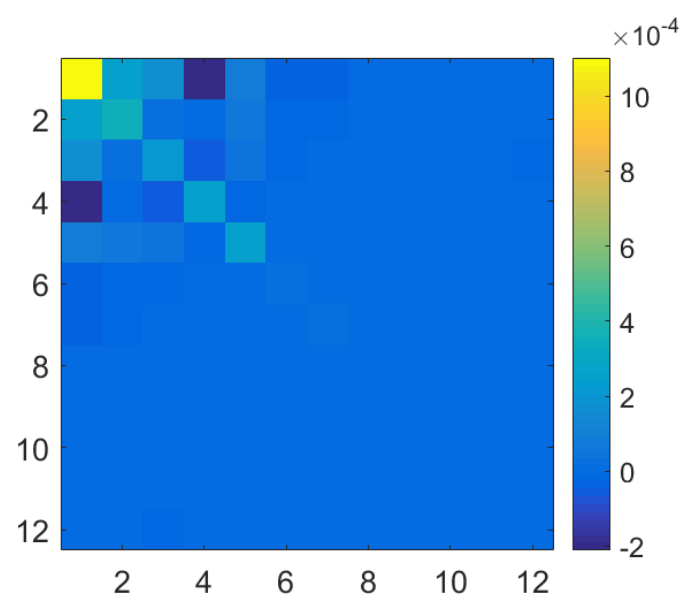

(a)

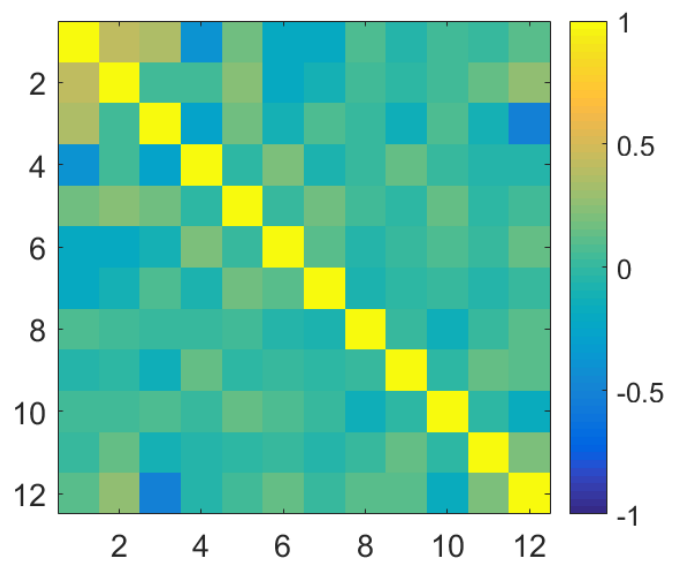

(b)

Figure 17: Covariance (a) and correlation (b) matrices for optimized instrumentation \#1 


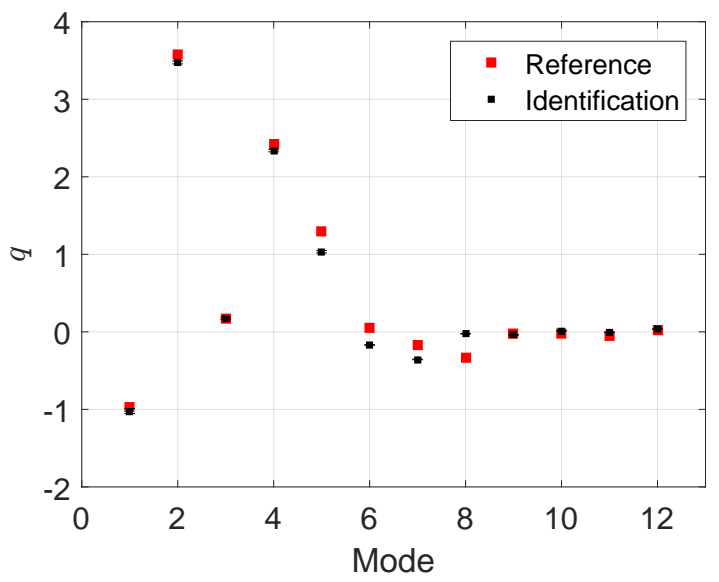

(a)

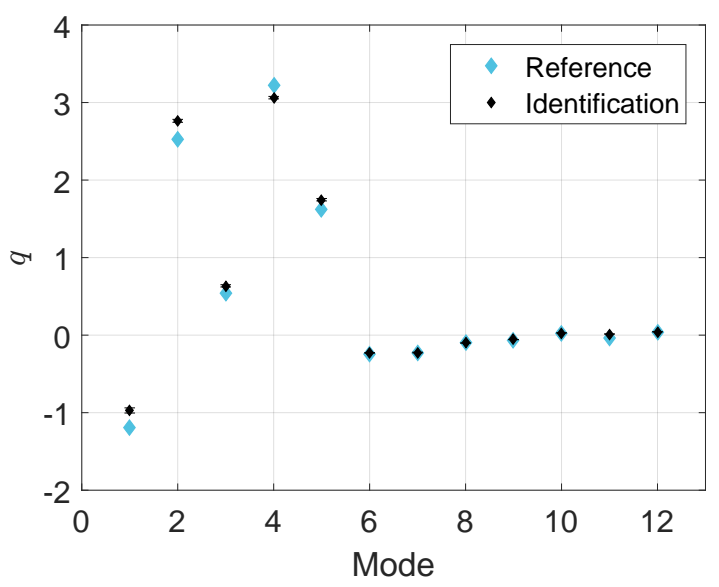

(b)

Figure 18: Comparison between reference and calibrated modal amplitudes for the inflation case of tires $A(a)$ and $B$ (b) using the optimized instrumentation \#1

The sequential exchange algorithm, which adds and deletes a gauge at each iteration, has also been used to determine an optimal instrumentation.

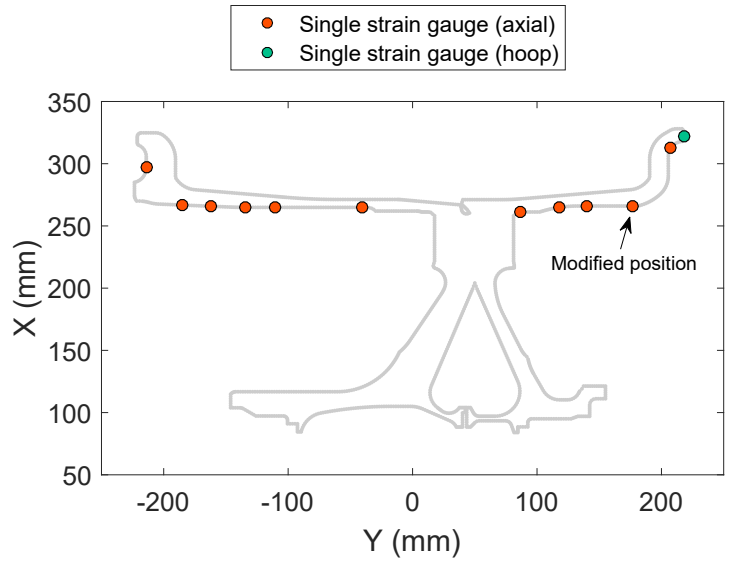

Figure 19: Locations of the 12 selected strain gauges for the optimized instrumentation \#2
However, it reveals very sensitive to the initial choice of the $M=12$ gauges required for its run. Therefore, several optimizations were performed using different initial sets of gauges randomly selected. A value of the information matrix determinant greater than that obtained with the optimized instrumentation \#1 was reached (i.e., $\log _{10}(\operatorname{det}([\boldsymbol{I}]))=80.54$ against 80.48 , representing a $14 \%$ change of the determinant value). The resulting instrumentation is illustrated in Figure 19 but differs only in one gauge on the outer wheel half whose new location is immediately adjacent to its previous position.

Last, the modified Fedorov algorithm was tested. The latter allows direct exchange to be performed between a gauge pair at each iteration. Whatever the chosen initial set of gauges, the algorithm demonstrates a great robustness and does not remain trapped into local minima far away from the global minimum. The value of the information matrix determinant was increased by $2 \%$ compared to the optimized instrumentation $\# 2$ (i.e., $\log _{10}(\operatorname{det}([\boldsymbol{I}]))=80.55$ against 80.54$)$. This value was not exceeded during all performed optimization steps, and the resulting instrumentation is shown in Figure 20. However, it is difficult to compare the performance of the different algorithms in more detail (e.g., computation time) because of the small size and linearity of the used FEM. Moreover, it may not be representative of more complex problems, for which the entire protocol should be revised.

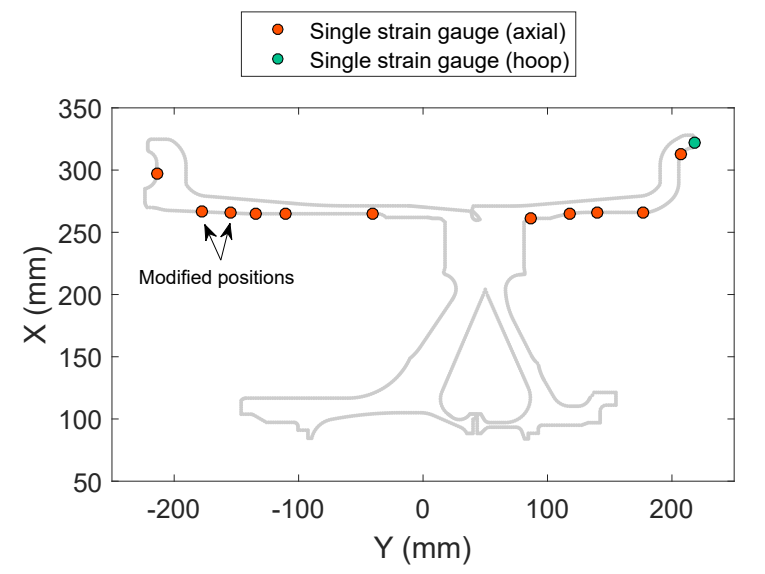

Figure 20: Locations of the 12 retained strain gauges for the optimized instrumentation \#3

Two strain gauges located on the inner wheel half have been slightly shifted compared to instrumentation \#2. Moreover, one can note that gauge locations in the bead seat area are similar to those of the standard instrumentation (Figure 8), particularly for the inner wheel half. The covariance and correlation matrices for this new instrumentation are shown in Figure 21 and are slightly changed compared to instrumentation \#1 (Figure 17). 


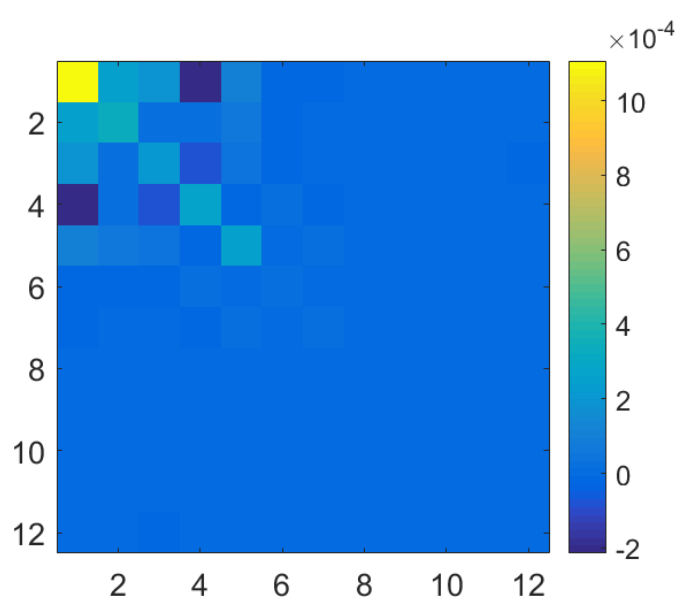

(a)

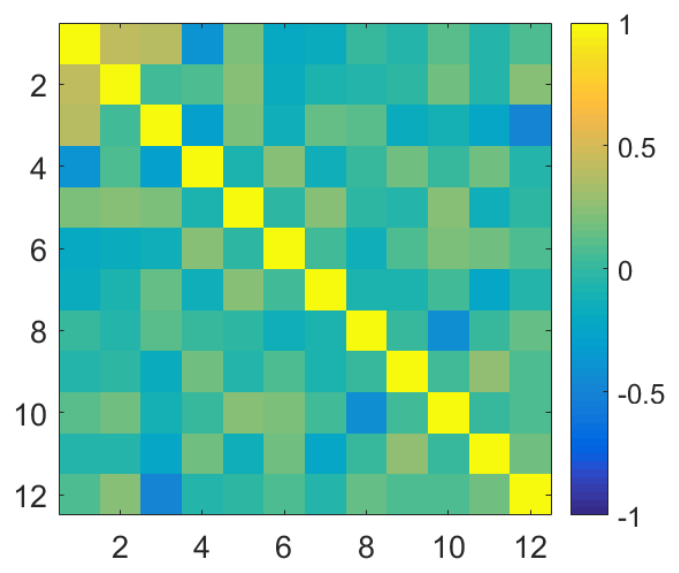

(b)

Figure 21: Covariance (a) and correlation (b) matrices for optimized instrumentation \#3

The instrumentation optimization based on the information matrix determinant maximization thus leads to the optimal placement of 12 strain gauges to calibrate at best the 12 loading parameters. It is now suggested to change the optimization criterion considering the direction of highest uncertainty and to assess the impact on the instrumentation.

The deletion procedure, for which the gauge with the lowest value of $f^{2}$ is removed, was used. The criterion change as a function of the iteration number is illustrated in Figure 22. The decrease of the criterion is slow until the 160th iteration. Then, some staircase profiles appear and characterize a rather sudden change in the direction of highest uncertainty. The criterion reaches an extremely low value (i.e., $\mathcal{I}_{\varphi}=3.7 \times 10^{-6}$ ) when $M=12$ gauges are kept, thereby indicating that the uncertainties of the sought parameters will be very high with the associated instrumentation. The latter should thus not be used to perform inverse identification of the loading parameters.

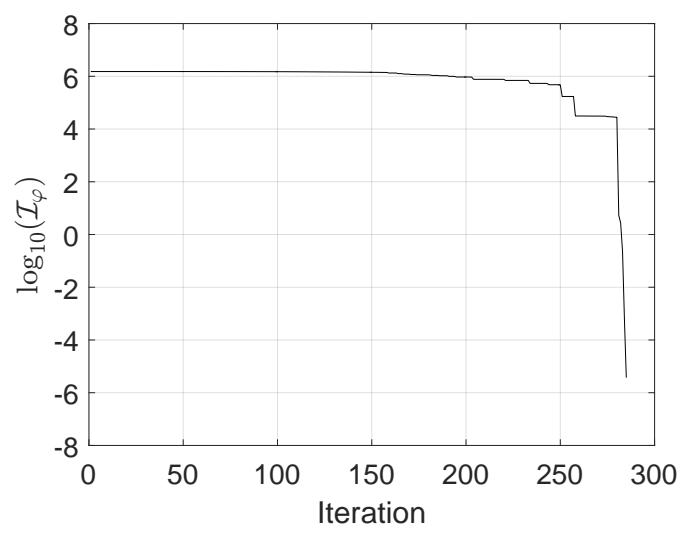

Figure 22: Lowest information matrix eigenvalue change with the number of iterations

An instrumentation made up of $M=17$ gauges is rather chosen to retain a reasonable value of the optimization criterion (i.e., $\mathcal{I}_{\varphi}=2.8 \times 10^{4}$ ). It corresponds to the last stage in Figure 22 and to the instrumentation shown in Figure 23.

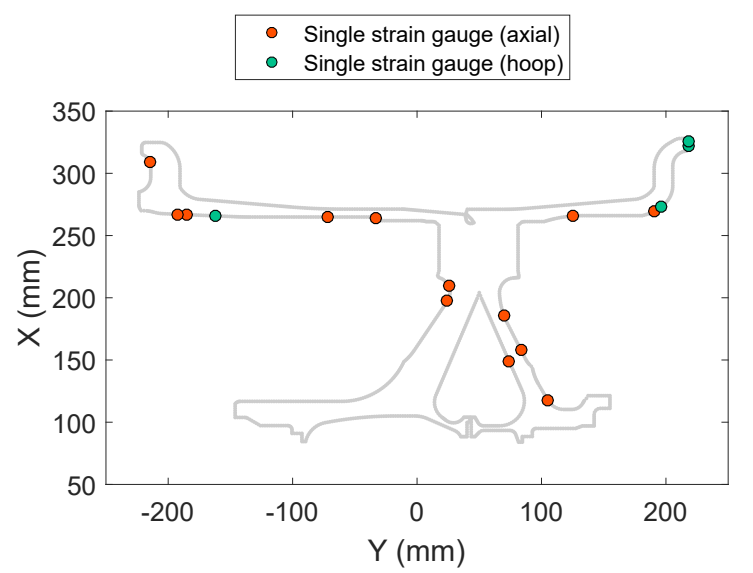

Figure 23: Location of the 17 strain gauges for optimization instrumentation \#4

This instrumentation is less intuitive than the previous ones obtained with the determinant criterion. Among the 17 gauges, 11 are located on the rim in a nonuniform way, and 6 in the web area. However, the latter ones are less sensitive to the local distribution of tire-rim interface loading than those located in the rim area (i.e., Saint-Venant's principle). It is thus surprising that they are retained in the instrumentation to identify loadings at the tire-rim interface. This result can be explained by a direction of highest uncertainty in the parameter space very far from the usual loadings applied to the wheel.

The covariance and correlation matrices obtained with this instrumentation are shown in Figure 23. The uncertainties are higher than before and this new instrumentation retains several correlations between the loading parameters. 


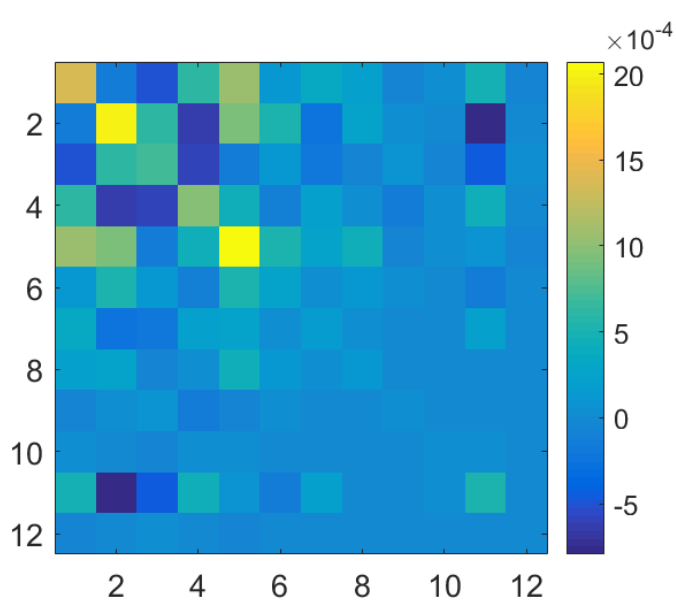

(a)

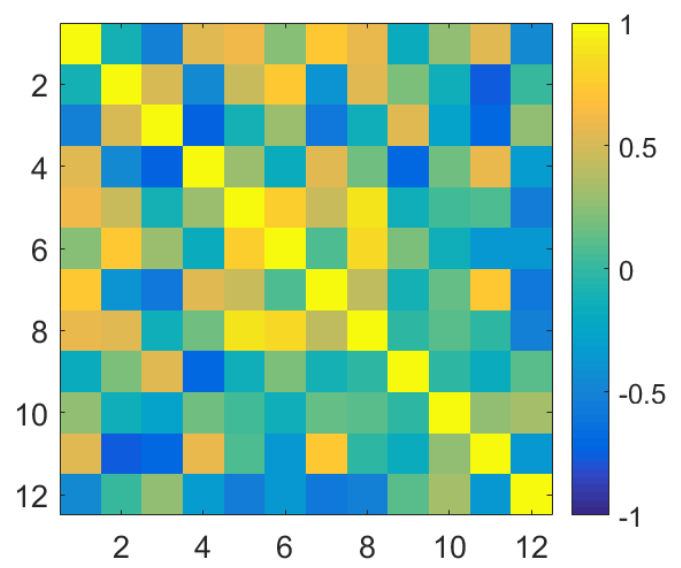

(b)

Figure 24: Covariance (a) and correlation (b) matrices for optimized instrumentation \#4

However, the calibrated modal amplitudes meet the reference values and the uncertainties remain acceptable (Figure 25). The error due to the truncation of the eigenmode basis to approximate tire-rim loadings is here again larger than the measurement uncertainty.

At this point, one may finally wonder which of the previous optimization protocols is the best. The results obtained for all of them are summarized in Table 2, where both the information matrix determinant and lowest eigenvalue are given for each optimized instrumentation.

\section{Table 2}

Optimization criteria values for each optimized instrumentation

\begin{tabular}{ccc}
\hline Optimized instrumentation & $\log _{10}(\operatorname{det}([\boldsymbol{I}]))$ & $\log _{10}\left(\mathcal{I}_{\varphi}\right)$ \\
\hline$\# 1$ & 80.48 & 4.896 \\
$\# 2$ & 80.54 & 4.895 \\
$\# 3$ & 80.55 & 4.894 \\
$\# 4$ (17 strain gauges) & 72.88 & 4.448 \\
\hline
\end{tabular}

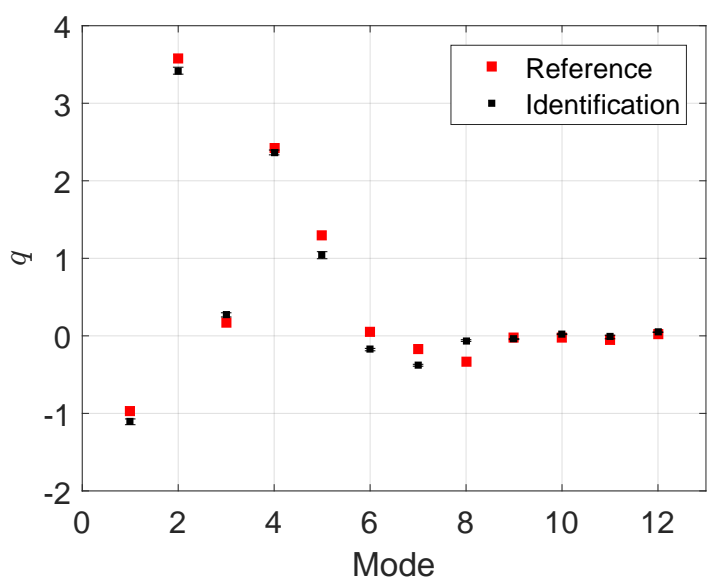

(a)

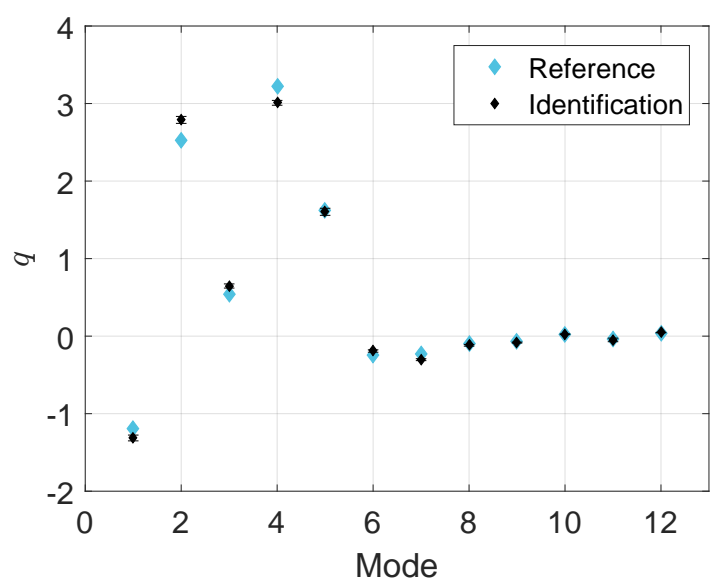

(b)

Figure 25: Comparison between reference and calibrated modal amplitudes for the inflation case of tires $A(a)$ and $B$ (b) using optimized instrumentation \#4

As a reminder, the results obtained considering the information matrix determinant as the optimization criterion show that instrumentation \#1 does not provide the best solution according to its own criterion, because the problem is intrinsically nonlinear and nonconvex. However, optimized instrumentations \#2 and \#3 give very similar results, without significant deterioration of lowest eigenvalue of the information matrix.

Each optimization algorithm is expected to lead to a "better" solution according to its own criterion. To validate this property, changes of the determinant and lowest eigenvalue of the information matrix with the number of iterations for protocols \#1 and \#4 (related to the respective instrumentations) are superimposed in Figure 26. The above expectation is indeed confirmed when the optimization is based on the maximization of the information matrix determinant (i.e., instrumentations \#1, \#2 and \#3), which achieves much higher values than when the lowest eigenvalue is considered as the optimization criterion (i.e., instrumentation \#4). Figure 26a shows that this difference between protocols \#1 and \#4 appears very early along the optimization process. However, 
more surprisingly, when the lowest eigenvalue of the information matrix is considered, as shown in Figure 26b, both procedures \#1 and \#4 provide very similar performances up to the 250-th iteration, with a slight advantage for the procedure \#4 focused on this specific target in the first 200 steps. However, after about 250 iterations, protocol \#4 suddenly diverges. This behavior can be traced to very abrupt discontinuities in the direction of highest uncertainty. These changes in the direction in which optimization is performed is responsible for the much more irregular behavior of procedure \#4 observed for both quantities of interest $\operatorname{det}([\boldsymbol{I}])$ and $\mathcal{I}_{\varphi}$. This is to be contrasted with procedure \#1 which considers all directions simultaneously, resulting in a much smoother behavior and hence a more robust character as well.

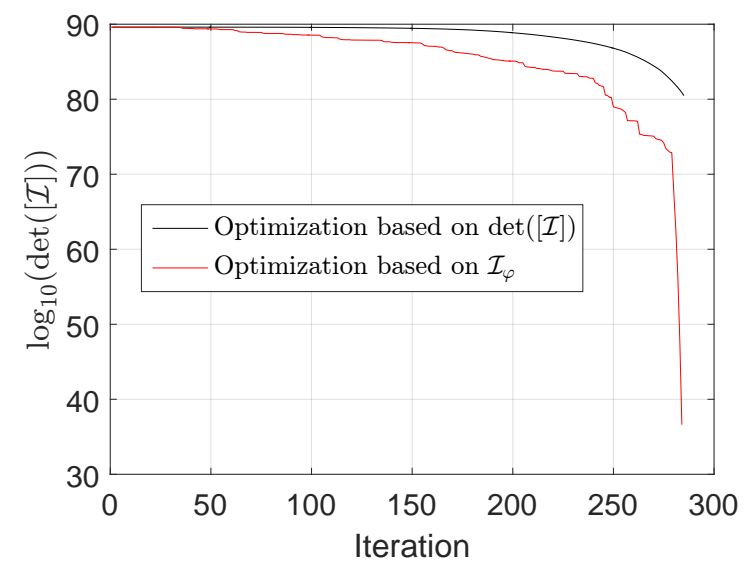

(a)

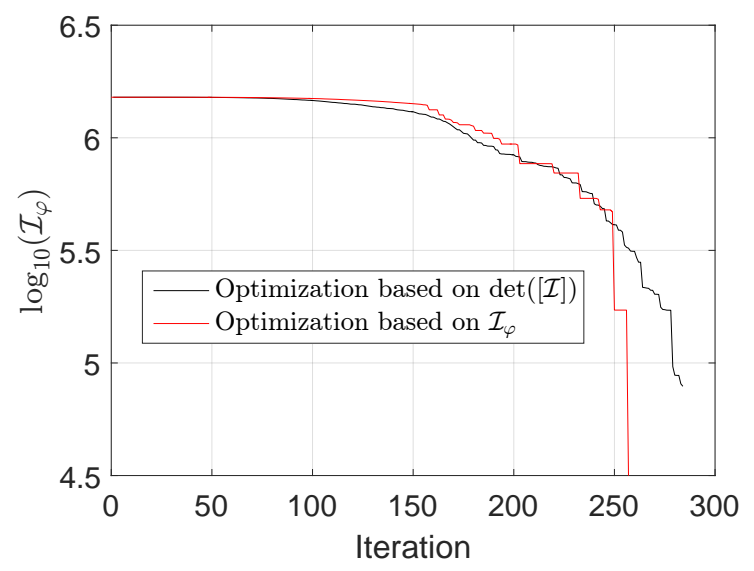

(b)

Figure 26: Changes of the determinant (a) and lowest eigenvalue (b) of the information matrix with the number of iterations for protocols \#1 and \#4

In the end, the procedure based on the lowest eigenvalue of the information matrix as the optimization criterion thus does not lead to a better instrumentation than those determined using the information matrix determinant (Table 2 and Figure 26). However, other optimization algorithms were not assessed for this criterion but may have improved the results.

\section{Conclusion}

The optimal sensor placement problem for tire-rim interface loading identification purposes was investigated. In conjunction with the optimal parameterization of tire-rim loading (Cosseron et al., 2019), it was proposed to optimize the instrumentation of an aircraft wheel to calibrate the loading parameters at best. The choice was made to only deal with strain gauge measurements, but the proposed framework can be extended to other types of sensor if needed. The aim was thus to determine the right number and location of sensors that minimize the uncertainties on the sought parameters.

To this end, optimization criteria and procedures, which were based on the information contained in the covariance matrix of the uncertainties affecting the loading parameter estimates, were used. In the context of tire-rim interface loading identification, the proposed methodology appears as a significant progress with respect to the state-of-art for two reasons: first, the loading parameter uncertainties were addressed (and very significantly reduced) while they are seldom mentioned in the literature; second, optimization procedures for the instrumentation were proposed to achieve a satisfactory configuration of strain gauges rather than relying on trials and errors or intuition.

Having detailed the inverse problem to solve to determine tire-rim interface loadings from experimental strain measurements, the identification of an inflation case was studied as a proof of concept considering a 2D-axisymmetric FEM of the wheel and two different tires. Two optimization criteria, namely, the determinant and the lowest eigenvalue of the information matrix, and several algorithms were then used to obtain the best estimates of the sought parameters. All optimized instrumentations led to drastically reduced uncertainties of the loading parameters. However, those obtained with the determinant criterion appeared to be more suitable to the studied problem. Therefore, any inflation loading applied to the wheel of a landing gear can be correctly calibrated with this kind of instrumentation.

The extension of the present framework to 3D loading cases (i.e., vertical and cornering) is necessary to obtain a complete answer to the problem of tire-rim loading identification. A similar work should thus be performed using 3D FEMs of the wheel. Shell elements could be used to mimic strain gauge surfaces. The post-processing of the strain tensor at element integration points would enable the search of the optimal strain gauge orientation to be automated, among other things. The impact of the FEM nonlinearities (e.g., contacts) should also be assessed. Furthermore, it would be interesting to enhance the optimization criterion to ensure robustness against sensor failures and mispositioning. Last, the framework may also be extended to the determination of other quantities of interest (e.g., for sizing purposes). The definition of an optimized gauging and the subsequent identification of tire-rim interface loadings for different conditions will allow the aircraft wheel manufacturer to conduct reliable fatigue analyses of the wheel and to predict its service life with a high degree of accuracy. 


\section{Funding Sources}

This work was funded by Safran Landing Systems and ANRT. It was part of a doctoral project under Industrial Convention of Formation by Research (CIFRE).

\section{Acknowledgments}

The authors acknowledge useful discussions with Prof. René Billardon, Senior Expert in Mechanics, from Safran Landing Systems site at Vélizy-Villacoublay, France.

\section{References}

Atkinson, A.C., 1982. Developments in the design of experiments. International Statistical Review 50, 161-177. doi:10.2307/1402599.

Atkinson, A.C., Donev, A.N., 1989. The construction of exact d-optimum experimental designs with application to blocking response surface designs. Biometrika 76, 515-526. doi:10.2307/2336117.

Balabin, I.V., Zubarev, N.A., 1964. Force interaction between a wide profile tire and the wheel rim. Automobil'naya Promyshlennost 30, 16-19.

Ballo, F., Gobbi, M., Mastinu, G., Previati, G., Zerboni, R., 2015. Motorcycle tire modeling, in: ASME 2015 International Design Engineering Technical Conferences and Computers and Information in Engineering Conference, Boston, Massachusetts, USA, ASME. doi:10.1115/ DETC2015-46607.

Ballo, F., Previati, G., Gobbi, M., Mastinu, G., 2017. A semi-analytical tyre model for the study of tyre/rim interaction on a road vehicle, in: ASME 2017 International Design Engineering Technical Conferences and Computers and Information in Engineering Conference, Cleveland, Ohio, USA, ASME. doi:10.1115/DETC2017-67730.

Ballo, F., Previati, G., Gobbi, M., Mastinu, G., 2018. Tire-rim interaction, a semi-analytical tire model. Journal of Mechanical Design 140. doi:10. 1115/1.4038927.

Bertin, M., Hild, F., Roux, S., Mathieu, F., Leclerc, H., Aimedieu, P., 2016. Integrated digital image correlation applied to elastoplastic identification in a biaxial experiment. Journal of Strain Analysis for Engineering Design 51, 118-131. doi:10.1177/0309324715614759.

Biderman, V.L., Guslitser, R.L., Sakharov, S.P., Nenakhov, B.V., Seleznev, I.I., Tsukerberg, S.M., 1969. Automotive tires, construction, design, testing and operation. NASA Technical Translation F-12, 382, NASA, Washington, District of Columbia, USA.

Chen, C.H.S., 1982. Prediction of bead area contact load at the tire-wheel interface using nastran, in: Tenth NASTRAN Users' Colloquium, New Orleans, Louisiana, USA, NASA. pp. 133-142.

Chiang, Y.J., Shih, C.D., Lin, C.C., Lee, H.K., Tseng, Y.Y., Tsai, K.C., Cheng, Y.H., 2000. Multi-variable effects on sealing pressure between tyres and rims. International Journal of Vehicle Design 23. doi:10.1504/ IJVD. 2000.001884.

Cook, R.D., Nachtsheim, C.J., 1980. A comparison of algorithms for constructing exact d-optimal designs. Technometrics 22, 315-324. doi:10.2307/1268315.

Cosseron, K., Gras, R., Mellé, D., Diebold, J.F., Hild, F., Roux, S., 2018. Inverse identification of the loading applied by a tire on a landing gear wheel, in: Residual Stress, Thermomechanics \& Infrared Imaging, Hybrid Techniques and Inverse Problems, Proceedings of the 2018 Annual Conference on Experimental and Applied Mechanics, Greenville, South Carolina, USA, Society for Experimental Mechanics, Springer, Cham. pp. 13-16. doi:10.1007/978-3-319-95074-7_3.

Cosseron, K., Mellé, D., Hild, F., Roux, S., 2019. Optimal parameterization of tire-rim interaction for aircraft wheels. Journal of Aircraft (accepté pour publication) doi:10.2514/1. c035343.

Curtiss, W.W., 1973. Principles of tire design. Tire Science and Technology 1, 77-98. doi:10.2346/1.2167156.

DuMouchel, W., Jones, B., 1994. A simple bayesian modification of doptimal designs to reduce dependence on a assumed model. Technometrics 36, 37-47. doi:10.1080/00401706.1994.10485399.
Dykstra, O., 1971. The augmentation of experimental data to maximize $|x ' x|$. Technometrics 13, 682-688. doi:10.2307/1267180.

European Aviation Safety Agency, 2010. Europen technical standard order (ETSO) C135a - Large aeroplane wheels and wheel and brake assemblies. European Aviation Safety Agency, Köln, Germany.

Federal Aviation Administration, 2008. Technical standard order (TSO) C135a - Transport airplane wheels and wheel and brake assemblies. Federal Aviation Administration, Washington, District of Columbia, USA.

Fedorov, V.V., 1972. Theory of optimal experiments. Academic Press, New York and London.

Fisher, R.A., 1935. The design of experiments. Oliver and Boyd, London and Edinburgh, United Kingdom.

Galil, Z., Kiefer, J., 1980. Time and space saving computer methods, related to mitchell's detmax, for finding d-optimum designs. Technometrics 22, 301-313. doi: $10.2307 / 1268314$.

Gras, R., Leclerc, H., Hild, F., Roux, S., Schneider, J., 2015c. Identification of a set of macroscopic elastic parameters in a $3 \mathrm{~d}$ woven composite: uncertainty analysis and regularization. International Journal of Solids and Structures 55, 2-16. doi:10.1016/j.ijsolstr.2013.12.023.

Gras, R., Mellé, D., Hild, F., 2015b. Loading identification on a tire/rim contact for an inflation pressure, in: 10th International Conference on Advances in Experimental Mechanics, Edingburgh, United Kingdom, British Society for Strain Measurement (BSSM).

Gupta, D.K., Dhingra, A.K., 2013. Input load identification from optimally placed strain gages using d-optimal design and model reduction. Mechanical Systems and Signal Processing 40, 556-570. doi:10.1016/j. ymssp. 2013.06.011.

Guyan, R.J., 1965. Reduction of stiffness and mass matrices. AIAA Journal 3, 380-380. doi:10.2514/3.2874.

Heinrietz, A., Lehrke, H.P., Rupp, A.N., Barthel, C., 2003. Identification of parametric tire models for the fatigue evaluation of suspension components. SAE Technical Paper Series 2003-01-1276 doi:10.4271/ 2003-01-1276.

Jansen, K.M.B., 1997. Effect of pressure on electrical resistance strain gages. Experimental Mechanics 37, 245-249. doi:10.1007/BF02317414.

Jeusette, J.P., Theves, M., 1992. Finite element analysis of tire/rim interface forces under braking and cornering loads. Tire Science and Technology 20, 83-105. doi:10.2346/1.2139512.

Johnson, M.E., Nachtsheim, C.J., 1983. Some guidelines for constructing exact d-optimal designs on convex design spaces. Technometrics 25 , 271-277. doi:10.1080/00401706.1983.10487880.

Kaipio, J., Somersalo, E., 2005. Statistical and computational inverse problems. Springer-Verlag New York, USA. doi:10.1007/b138659.

Kammer, D.C., 1991. Sensor placement for on-orbit modal identification and correlation of large space structures. Journal of Guidance, Control, and Dynamics 14, 251-259. doi:10.2514/3. 20635.

Kammer, D.C., Tinker, M.L., 2004. Optimal placement of triaxial accelerometers for modal vibration tests. Mechanical Systems and Signal Processing 18, 29-41. doi:10.1016/S0888-3270(03)00017-7.

Kandarpa, S., Spencer, B.F., Kirkner, D.J., Champion, M., 1994. Determination of tire-wheel interface loads for aircraft wheels. Journal of Aircraft 31, 433-440. doi:10.2514/3.46504.

Kavanagh, K.T., Clough, R.W., 1971. Finite element applications in the characterization of elastic solids. International Journal of Solids and Structures 7, 11-23. doi:10.1016/0020-7683(71)90015-1.

Kiefer, J., 1959. Optimum experimental designs. Journal of the Royal Statistical Society. Series B (Methodological) 21, 272-319.

Kiefer, J., Wolfowitz, J., 1960. The equivalence of two extremum problems. Canadian Journal of Mathematics 12, 363-366. doi:10.4153/ CJM-1960-030-4.

Kirkegaard, P.H., 1991. Optimal design of experiments for parametric identification of civil engineering structures. Ph.D. thesis. Aalborg University, Denmark.

Kirkner, D.J., Spencer, B.F., Schudt, E.E., Kandarpa, S., Chawla, M.D., 1993. Determination of tire-wheel interface pressure distribution for aircraft wheels, in: 34th Structures, Structural Dynamics and Materials Conference, La Jolla, California, USA, American Institute of Aeronautics and Astronautics (AIAA). pp. 415-423. doi:10.2514/6.1993-1343. 
Masroor, S.A., Zachary, L.W., 1991. Designing an all-purpose force transducer. Experimental Mechanics 31, 33-35. doi:10.1007/BF02325720.

Mehra, R., 1974. Optimal inputs for linear system identification. IEEE Transactions on Automatic Control 19, 192-200. doi:10.1109/TAC. 1974. 1100554.

Meng, J., Zhu, P., Ji, Q.H., Liu, Z., 2012. An improved pressure distribution model of automotive wheel's radial fatigue. Applied Mechanics and Materials 201-202, 287-291. doi:10.4028/www. scientific.net/AMM. 201-202.287.

Meo, M., Zumpano, G., 2005. On the optimal sensor placement techniques for a bridge structure. Engineering Structures 27, 1488-1497. doi:10. 1016/j. engstruct. 2005.03.015.

Meyer, R.K., Nachtsheim, C.J., 1995. The coordinate-exchange algorithm for constructing exact optimal experimental designs. Technometrics 37 , 60-69. doi:10.2307/1269153.

Mitchell, T.J., 1974. An algorithm for the construction of "d-optimal" experimental designs. Technometrics 16, 203-210. doi:10.2307/1267940.

Morelli, E.A., Klein, V., 1990. Optimal input design for aircraft parameter estimation using dynamic programming principles, in: AIAA Atmospheric Flight Mechanics Conference, Portland, Oregon, USA, American Institute of Aeronautics and Astronautics (AIAA).

Nguyen, T., Schonning, A., Eason, P., Nicholson, D., 2012. Methods for analyzing nose gear during landing using structural finite element analysis. Journal of Aircraft 49, 275-280. doi:10.2514/1. c031519.

Pelle, R.G., 1994. Fem simulation of tire/rim seating process. Tire and Science Technology 22, 76-98. doi:10.2346/1.2139537.

Roux, S., Hild, F., 2020. Optimal procedure for the identification of constitutive parameters from experimentally measured displacement fields. International Journal of Solids and Structures 184, 14-23. doi:10.1016/ j.ijsolstr.2018.11.008.

Rupp, A.N., Heinrietz, A., Ehl, O., 2002. Simulation of the experimental proof out of wheels and hubs. SAE Technical Paper Series 2002-01-1202 doi:10.4271/2002-01-1202.

Schmaltz, S., Willner, K., 2014. Comparison of different biaxial tests for the inverse identification of sheet steel material parameters. Strain 50, 389-403. doi:10.1111/str. 12080.

Schudt, E.E., Kirkner, D.J., Spencer, B.F., Chawla, M.D., 1994. Threedimensional verification of an axisymmetric algorithm for tire-wheel interface load recovery. SAE Technical Paper 941176 doi:10.4271/941176.

Shah, P.C., Udwadia, F.E., 1977. A methodology for optimal sensor locations for identification of dynamic systems. Journal of Applied Mechanics 45, 188-196. doi:10.1115/1.3424225.

Sherwood, J.A., Ayres, J.M., Gross, T.S., Watt, D.W., 1995a. An investigation of tire-wheel interface loads using adina. Computers and structures 56, 377-387. doi:10.1016/0045-7949(95)00030-K.

Sherwood, J.A., Fussell, B.K., Edwards, W.R., Gross, T.S., Watt, D.W., 1995b. Study of the pressure distribution on an aircraft tire-wheel interface. Journal of Aircraft 32, 921-928. doi:10.2514/3.46819.

Spencer, B.F., Kirkner, D.J., Schudt, E.E., Chawla, M., Homa, D., 1995. Experimental verification of an algorithm for determination of tirewheel interface loads. SAE Technical Paper 951435 doi:10. 4271/951435.

Spencer, B.F., Kirkner, D.J., Schudt, E.E., Kandarpa, S., 1993. Aircraft life wheel assessment. Technical Report. University of Notre Dame, Indiana, USA.

Stearns, J.C., Srivatsan, T.S., Gao, X., Lam, P.C., 2006. Understanding the influence of pressure and radial loads on stress and displacement response of a rotating body: the automobile wheel. International Journal of Rotating Machinery 2006. doi:10.1155/IJRM/2006/60193.

Tamarozzi, T., Risaliti, E., Rottiers, W., Janssens, K., Desmet, W., 2016. Noise, ill-conditioning and sensor placement analysis for force estimation through virtual sensing, in: International Conference on Noise and Vibration Engineering, Katholieke Univ Leuven, Dept Werktuigkunde. pp. 1741-1756.

Tseng, N.T., Pelle, R.G., Chang, J.P., 1989. Numerical simulation for the tire-rim interface for a radial passenger tire. SAE Technical Paper Series 890641 doi:10.4271/890641.

Van Schalkwyk, D.J., 1971. On the design of mixture experiments. Ph.D. thesis. University of London, United Kingdom.
Vitse, M., Poncelet, M., Iskef, A., Dufour, J.E., Gras, R., Bouterf, A., Raka, B., Giry, C., Gatuingt, F., Hild, F., Ragueneau, F., Roux, S., 2020. Toward virtual design and optimization of a structural test monitored by a multi-view system. Journal of Strain Analysis for Engineering Design in press. doi:10.1177/0309324720910887.

Walter, J.D., Kiminecz, R.K., 1975. Bead contact pressure measurements at the tire-rim interface. SAE Technical Paper Series 750458 doi:10.4271/ 750458.

Wan, X., Shan, Y., Liu, X., He, T., Wang, J., 2017. Tire-rim interface pressure of a commercial vehicle wheel under radial loads: theory and experiment. Measurement Science and Technology 28, 1-9. doi:10. 1088/1361-6501/aa8895.

Wan, X., Shan, Y., Liu, X., Wang, H., Wang, J., 2016. Simulation of biaxial wheel test and fatigue life estimation considering the influence of tire and wheel camber. Advances in Engineering Software 92, 57-64. doi:10. 1016/j. advengsoft. 2015.11.005.

Wickham, M.J., Riley, D.R., Nachtsheim, C.J., 1995. Integrating optimal experimental design into the design of a multi-axis load transducer. Journal of Engineering for Industry 117, 400-405. doi:10.1115/1.2804346.

Wynn, H.P., 1970. The sequential generation of d-optimum experimental designs. The Annals of Mathematical Statistics 41, 1655-1664. doi:10. 1214/aoms/1177696809.

Zaharov, S.P., 1957. Feasibility study of how to make compression measurements of the tire bead against the rim. Kauchuk i Rezina 16, 30-31. 